\title{
Early inpatient rehabilitation for acutely hospitalized older patients: a systematic review of outcome measures
}

\author{
${\text { Patrick Heldmann }{ }^{*} \text { (D), Christian Werner }}^{2,3} \mathbb{E}$, Nacera Belala ${ }^{1}$, Jürgen M. Bauer ${ }^{2,3}$ and Klaus Hauer ${ }^{2}$
}

\begin{abstract}
Background: Selecting appropriate outcome measures for vulnerable, multimorbid, older patients with acute and chronic impairments poses specific challenges, which may have caused inconsistent findings of previous intervention trials on early inpatient rehabilitation in acutely hospitalized older patients. The aim of this review was to describe primary outcome measures that have been used in randomized controlled trials (RCTs) on early rehabilitation in acutely hospitalized older patients, to analyze their matching, and to evaluate the effects of matching on the main findings of these RCTs.

Methods: A systematic literature search was conducted in PubMed, Cochrane CENTRAL, CINAHL, and PEDro databases. Additional studies were identified through reference and citation tracking. Inclusion criteria were: RCT, patients aged $\geq 65$ years, admission to an acute hospital medical ward (but not to an intensive medical care unit), physical exercise intervention (also as part of multidisciplinary programs), and primary outcome measure during hospitalization. Two independent reviewers extracted the data, assessed the methodological quality, and analyzed the matching of primary outcome measures to the intervention, study sample, and setting. Main study findings were related to the results of the matching procedure.
\end{abstract}

Results: Twenty-eight articles reporting on 24 studies were included. A total of 33 different primary outcome measures were identified, which were grouped into six categories: functional status, mobility status, hospital outcomes, adverse clinical events, psychological status, and cognitive functioning. Outcome measures differed considerably within each category and showed a large heterogeneity in their matching to the intervention, study sample, and setting. Outcome measures that specifically matched the intervention contents were more likely to document intervention-induced benefits. Mobility instruments seemed to be the most sensitive outcome measures to reveal such benefits.

Conclusions: This review highlights that the selection of outcome measures has to be highly specific to the intervention contents as this is a key factor to reveal benefits attributable to early rehabilitation in acutely hospitalized older patients. Inappropriate selection of outcome measures may represent a major cause of inconsistent findings reported on the effectiveness of early rehabilitation in this setting.

Trial registration: PROSPERO CRD42017063978.

Keywords: Acute care, Hospitalization, Aged, Rehabilitation, Exercise, Outcome measures

\footnotetext{
* Correspondence: heldmann@nar.uni-heidelberg.de

${ }^{1}$ Network Aging Research (NAR), Heidelberg University, Bergheimer Str. 20,

69115 Heidelberg, Germany

Full list of author information is available at the end of the article
}

(c) The Author(s). 2019 Open Access This article is distributed under the terms of the Creative Commons Attribution 4.0 International License (http://creativecommons.org/licenses/by/4.0/), which permits unrestricted use, distribution, and reproduction in any medium, provided you give appropriate credit to the original author(s) and the source, provide a link to the Creative Commons license, and indicate if changes were made. The Creative Commons Public Domain Dedication waiver (http://creativecommons.org/publicdomain/zero/1.0/) applies to the data made available in this article, unless otherwise stated. 


\section{Background}

Older patients treated in hospital - and those who treat them - face complex challenges which arise from a multitude of negative health conditions. In addition to acute medical illness as the cause of the hospital admission and the high prevalence of multimorbidity in this patient population, older patients frequently show further associated geriatric conditions, such as malnutrition, cognitive impairment, delirium, impairments in (instrumental) activities of daily living ([I]ADL), incontinence, and sensory impairment [1]. Apart from the fact that each of these conditions will request a specific, often enough individualized response, the mass of negative conditions, and the advanced frailty status frequently observed in these patients, put them at an extraordinary risk for hospital-associated deconditioning. As an expected consequence, the prevalence of functional decline during hospital stay is high, varying from 30 to $80 \%$ depending on the assessment methodology, medical status, and age cohorts included [2,3]. The consequences of this decline during are manifold, ranging from re-hospitalization, nursing home placement [4], and subsequent mortality [5] to an increased number of falls, poor quality of life, and increased use of healthrelated resources [6].

For all patients admitted to acute medical care, the subsequent phase of immobilization is crucial as it will drastically impair their functional status to a level where autonomy is seriously endangered [7]. Consequently, hospital admission represents a vulnerable period in the treatment process in which an early onset of rehabilitation and physical training is of utmost importance, providing the basis for post-recovery and subsequent therapeutic and rehabilitative care.

The effect of early physical exercise interventions in acutely hospitalized older patients has already been examined in a number of previous systematic reviews [3, 8-13], reporting heterogeneous results across different outcomes and outcome categories such as hospital outcomes, adverse clinical events, or functional and mobility outcomes. A potential cause of this inconclusive evidence for the benefits of early physical exercise interventions has been addressed in one of these reviews, hypothesizing that the adaption level of the intervention to the capabilities of the patients might have played a critical role for the effectiveness of such interventions in acutely hospitalized older patients [13]. However, contrary to this hypothesis, patient-tailored physical exercise interventions were not found to be superior to those interventions that were not. Another potential cause for the still limited evidence might be the use of various outcome measures, which has been reported in most of the aforementioned reviews [3, 10, 11, 13]. However, none of these reviews specifically addressed the heterogeneity and the appropriateness of the outcome measures selected in the previous studies. The selection of the outcome measure(s), i.e. the operationalization of the outcome, is a critical step in designing a valid and useful clinical study [14]. In absence of an appropriate outcome measure, the impact of an intervention may be lost and benefits of the intervention may not be captured $[14,15]$. Outcome measures used in clinical trials seem to have been most frequently evaluated focusing only on their psychometric properties $[16,17]$. However, such focus fails to address also important questions about the suitability of the measures for their intended use. When reviewing and selecting an appropriate outcome measure for a tailored study design, the evaluation of the psychometric properties represents a first step, but also further requirements have to be considered. Most importantly, researchers should select outcome measures that match the intervention contents and specifically address the areas being targeted by them. If an intervention content is not well represented in the outcome measure, true changes in the relevant areas the researches expect to be influenced by the specific intervention may be lost because the selected outcome measure was unable to capture it. Further, it is important to determine whether the outcome measures are feasible in the target population. Feasibility aspects such as floor effects, indicating an overtaxation of patients, and ceiling effects, indicating an insufficient test challenge, must be considered, especially in the acute hospital setting with a highly heterogeneous patient population. Another criterion that must be considered when selecting appropriate outcome measures is to determine whether any features of the items could be problematic for use in the research setting. For example, IADL scales include items that assess an individual's ability to perform instrumental home or community activities such as housekeeping and going shopping, which cannot be appropriately assessed within the acute care hospital setting $[14,18]$. Meeting these requirements in the early hospital-based geriatric rehabilitation poses a particular challenge based on the fact, that acutely hospitalized older patients represent a heterogeneous, multimorbid and vulnerable patient population in a complex environment during a critical phase of recovery [9]. Consequently, potential multiple goals in the treatment of these patients will go along with different intervention strategies and outcome measures to be amalgamated into a specifically tailored study design, which may not have been achieved in previous studies.

The aim of this systematic review was (1) to describe outcome measures as used in previous intervention trials for early rehabilitation in acutely hospitalized older patients and analyze their matching to the contents of the intervention, the study sample, and the acute care 
hospital setting, and (2) to evaluate the effects of matching on the main findings reported in these intervention trials.

\section{Methods}

\section{Search strategy and study selection}

A systematic literature search was conducted in the electronic databases of PubMed, Cochrane CENTRAL, CINAHL, and PEDro from inception to December 2016. An extensive search strategy was developed for the PubMed database (Additional file 1: Table S1) and adjusted to the other electronic databases. Manual searching was also performed to identify additional studies by scanning reference lists of relevant review articles and included articles.

The inclusion criteria were as follows: (1) randomized, controlled intervention trial (RCT), (2) in older people aged 65 years or older (or 95\% of participants aged at least 65 years), (3) admitted to an acute hospital medical ward but (4) not to an intensive medical care unit, (5) with a physical exercise intervention or a multidisciplinary program with physical exercise as a training component, both performed in an acute hospital medical ward, and (6) at least one primary outcome measure during acute care hospitalization. Studies were excluded if they were conducted in subacute hospital settings (e.g. rehabilitation wards), feasibility studies, or written in languages other than English.

The selection process was conducted following the methodology as described in the method guidelines of the Cochrane Collaboration [19]. Each step of the selection process was performed independently by two researchers $(\mathrm{PH}, \mathrm{NB})$, and disagreements were resolved by consensus or third party consultation (KH, JMB). The review followed the PRISMA guidelines for reporting systematic reviews and meta-analyses (see Additional file 2 for the completed PRISMA checklist [20]) and was registered at the PROSPERO International prospective register of systematic reviews (PROSPERO 2017: CRD42017063978).

\section{Data extraction}

Data extraction was completed by the two reviewers $(\mathrm{PH}, \mathrm{NB})$ using a standardized data collection form as recommended by the Cochrane Collaboration [21]. For each study, the following data were extracted: author, country, sample characteristics, primary and secondary outcome measures during hospitalization, time point of measurement, intervention contents, and main findings on primary outcome measures. The extracted data were structured into a table and systematically analyzed.

\section{Data analysis}

\section{Matching of outcome measures}

An initial set of guidelines to help evaluate the matching of outcome measures for clinical trials have been proposed by Coster (2013) [14]. Taking these guidelines into account, the primary outcome measures identified for each study during hospitalization were matched with the intervention contents, the sample included in the study, and the acute care hospital setting. The criteria used for this matching procedure were provided in Table 1 . The matching procedure was performed independently by two researchers (PH, CW), and any disagreements were resolved by consensus or third party consultation (KH, JMB).

The main findings reported on the primary outcomes were subsequently related to the results of the matching procedure, with special focus on the matches between the outcome measures and the intervention contents, representing the most important factor to demonstrate the impact on the relevant areas being targeted by an intervention [14]. The evaluation of the intervention effects was based on the significance level of betweengroup differences in the primary outcomes. $P$-values $\leq 0.05$ were considered statistically significant.

\section{Quality rating}

Each included study was assessed using the PEDro scale, which consists of 11 items for rating the methodological quality of RCTs [23]. When available, confirmed PEDro scores from the PEDro database were used for the quality rating [24]. If no confirmed PEDro score was available, the quality rating was performed independently by two researchers $(\mathrm{PH}, \mathrm{NB})$. Disagreements were resolved by consensus or third party consultation (KH, JMB). A study with a PEDro score of $\leq 5$ points is considered to be of low methodological quality at high risk of bias [25].

\section{Results}

The search strategy yielded 17.074 potentially relevant articles (Fig. 1). After removing duplicates and screening of title and/or abstract, 184 articles were obtained in full text and evaluated for eligibility based on the predefined inclusion criteria. In total, 28 articles published between 1995 and 2016 were identified for inclusion. As four [26-29] and another two included articles [30, 31] reported each on the same RCT, the search finally resulted in 24 identified studies. The detailed data extracted for each of these studies were presented in Table 2.

\section{Methodological quality}

Total PEDro scores ranged from 2 to 8 points, with a mean score of $6.0 \pm 1.7$ points. High methodological quality and low risk of bias were found for 17 studies (70.8\%), with a PEDro score of $>5$ points $[27,31,32,34$, $39-46,48,49,51-53]$. Seven studies (29.2\%) did not exceed a score of 5 points, indicating a low methodological quality and high risk of bias [33, 35-37, 47, 50, 54]. The 
Table 1 Criteria for the matching of an outcome measure with the intervention, study sample, and setting

\begin{tabular}{|c|c|c|c|}
\hline \multicolumn{2}{|l|}{ Criteria } & \multicolumn{2}{|l|}{ Rating } \\
\hline Intervention & $\begin{array}{l}\text { Did the outcome measure match an } \\
\text { intervention content? }\end{array}$ & "Match" & $\begin{array}{l}\text { The outcome measure specifically addressed the exercise intervention or an } \\
\text { intervention content of the multidisciplinary program (e.g., } 6 \text {-Meter Walking } \\
\text { Test } \rightarrow \text { treadmill walking training; discharge destination } \rightarrow \text { discharge } \\
\text { planning). }\end{array}$ \\
\hline & & $\begin{array}{l}\text { "Limited } \\
\text { match" }\end{array}$ & $\begin{array}{l}\text { The outcome measure addressed the exercise intervention or an intervention } \\
\text { content of the multidisciplinary program only to a limited extent and/or } \\
\text { included only single items that specially matched to the intervention (e.g., } \\
\text { Barthel Index [transfer, mobility, and stairs items] } \rightarrow \text { strengthening and } \\
\text { mobility exercises; physical activity monitoring } \rightarrow \text { weight-bearing exercises) }\end{array}$ \\
\hline & & $\begin{array}{l}\text { "No } \\
\text { match" }\end{array}$ & $\begin{array}{l}\text { The outcome measure did not directly address the exercise intervention or an } \\
\text { intervention content of the multidisciplinary program or the construct of the } \\
\text { outcome measure was not addressed in the intervention (e.g., Lawton IADL } \\
\text { scale } \rightarrow \text { no IADL training content or discharge destination } \rightarrow \text { additional } \\
\text { exercise intervention). }\end{array}$ \\
\hline \multirow[t]{4}{*}{$\begin{array}{l}\text { Study } \\
\text { sample }\end{array}$} & $\begin{array}{l}\text { Was the outcome measure feasible in the } \\
\text { study sample? }\end{array}$ & "Match" & $\begin{array}{l}\text { The outcome measure showed no floor or ceiling effects (continuous } \\
\text { outcomes) or represented no rare event (dichotomous outcomes). Ceiling and } \\
\text { floor effects were defined as }(1) \geq 15 \% \text { of participants reaching a score within } \\
\text { the best or worst } 15 \% \text { of the instrument's rating scale [22] or (2) when the } \\
\text { mean score of the sample was within the best or worst 15\% of the rating } \\
\text { scale. Rare events were defined when the incidence of a dichotomous } \\
\text { outcome (e.g., falls, mortality) was } \leq 15 \% \text { in the sample. }\end{array}$ \\
\hline & & $\begin{array}{l}\text { "No } \\
\text { match" }\end{array}$ & $\begin{array}{l}\text { The outcome measure showed floor or ceiling effects (continuous outcomes) } \\
\text { or represented a rare event (dichotomous outcomes). }\end{array}$ \\
\hline & $\begin{array}{l}\text { How high was the missing data rate for the } \\
\text { outcome measure in the study sample? }\end{array}$ & "Match" & $\begin{array}{l}\text { The outcome measure had an acceptable missing data rate. Missing data } \\
\text { included any outcome data that (1) could not be collected for reasons other } \\
\text { than death or study withdrawal or (2) were collected but not presented. A } \\
\text { missing data rate of }<15 \% \text { was considered as acceptable. }\end{array}$ \\
\hline & & $\begin{array}{l}\text { "No } \\
\text { match" }\end{array}$ & The outcome measure did not have an acceptable missing data rate ( $\geq 15 \%)$. \\
\hline \multirow[t]{3}{*}{ Setting } & $\begin{array}{l}\text { Did the outcome measure match the acute } \\
\text { care hospital setting? }\end{array}$ & "Match" & $\begin{array}{l}\text { The outcome measure addressed a construct or activities that can be } \\
\text { appropriately assessed within the acute care hospital setting (e.g., hospital } \\
\text { costs or Barthel Index). }\end{array}$ \\
\hline & & $\begin{array}{l}\text { "Limited } \\
\text { match" }\end{array}$ & $\begin{array}{l}\text { The outcome measure addressed a construct or activities that can be } \\
\text { appropriately assessed only to a limited extent within the acute care hospital } \\
\text { setting and/or included only single items or contents that were appropriate } \\
\text { for use within the acute care hospital setting (e.g., combined ADL-IADL } \\
\text { measures). }\end{array}$ \\
\hline & & $\begin{array}{l}\text { "No } \\
\text { match" }\end{array}$ & $\begin{array}{l}\text { The outcome measure addressed a construct or activities that cannot be } \\
\text { appropriately assessed within the acute care hospital setting (e.g., IADL } \\
\text { measures). }\end{array}$ \\
\hline
\end{tabular}

detailed quality scores on the PEDro scale for each RCT are provided in Additional file 3: Table S2.

\section{Study samples}

The mean sample size was $357 \pm 421$ and varied considerably from 15 [36] to 1632 [47] participants, with half of the studies $(n=12,50.0 \%)$ recruiting at least 200 participants [30, 32, 39, 42, 44, 46-51, 53]. Participants' age across studies averaged $80.0 \pm 3.4$, with a range from 71 [38] to 85 [33] years. Identified studies predominantly included older patients with general medical conditions $(n=12,50.0 \%)[32-34,36,39,40,42,44,46-48,50]$ or acute hip fracture $(n=8,33.3 \%)[27,30,37,41,43,49$, $51,53]$. Other patient characteristics for study inclusion were acute exacerbation of chronic obstructive pulmonary disease (COPD) $(n=2,8.3 \%)$ [38, 45], delirium $(n=1,4.2 \%)$ [52], or abdominal surgery $(n=1$, $4.2 \%)$ [35].

\section{Interventions}

Early inpatient rehabilitation interventions could basically be divided into two categories: (1) "hospital usual care" with an additional or modified exercise program as included in 14 studies [32-45] or (2) multidisciplinary programs with an exercise component as included in 10 studies [27, 30, 46-53]. In the following, we refer to these two categories as exercise interventions and multidisciplinary programs, respectively.

Multidisciplinary intervention teams usually consisted of geriatricians, nurses, physical therapists, occupational therapists, dieticians, and/or social workers. Apart from 


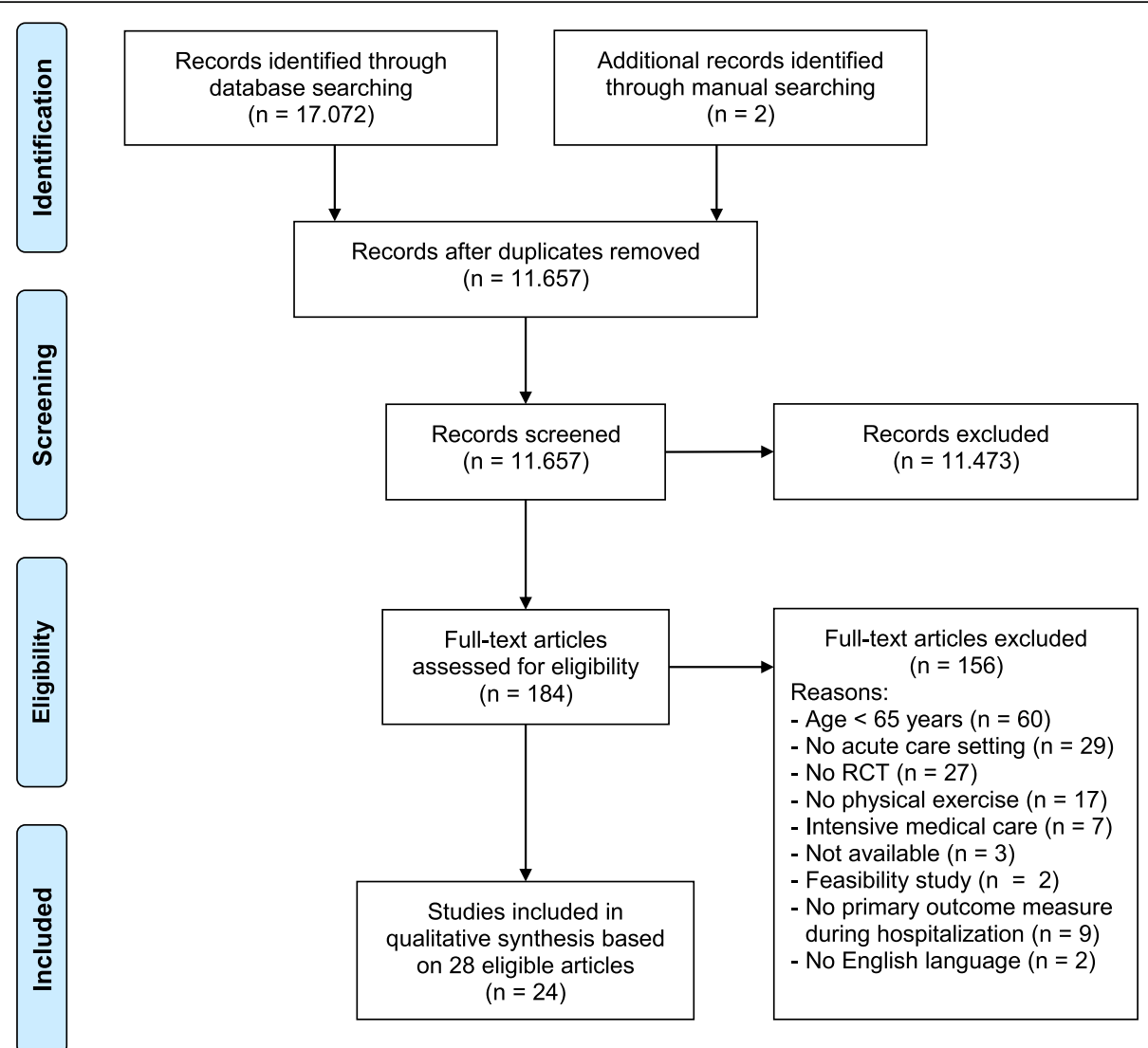

Fig. 1 PRISMA flow chart of study selection

the exercise component, multidisciplinary programs included components of comprehensive geriatric assessment $[27,30,46-50,52,53]$, multidisciplinary team meetings and individual care planning [27, 30, 46-51, 53], discharge planning [30, 46-53], nutritional interventions [27, 30, 47, 48, 50, 52], prevention and treatment of complications (e.g., vitamin supplementation, screening of infections) [27, 51], cognitive interventions [26, $47,48,50,52]$, psychological interventions [47, 48, 50, $52]$, staff education [27, 51], or specifically-designed environments $[47,48,50]$.

The content of the exercise component of the multidisciplinary programs most frequently included ADL training [27, 30, 47-51] and/or strength training [27, 30, 51]. Three studies did not provide detailed information on the content of the exercise component apart from stating that it included physical and/or occupational therapy [46, 52, 53].

Exercise interventions were usually supervised by physiotherapists, occupational therapists, nurses, allied health assistants, or staff specifically trained by physiotherapists. Intervention contents included modified or additional exercises with walking training [36, 40-44], strength training [33, 39-41, 44], ADL training [32, 36,
$37,42]$, flexibility training $[38,44]$, lower-limb endurance training [38, 45], cognitive exercises [32, 39], balance training [40], transfer training [40, 41], physical activity (PA) behavior intervention [34, 38], IADL training [36], breathing exercises [38], and/or proprioceptive neuromuscular facilitation exercises [35].

Participants in the control groups of the studies generally received usual care according to the general routines of the hospital they were admitted to.

\section{Outcome measures}

Identified outcome measures varied considerably among the included studies, with a total of 33 different primary outcome measures. They can be grouped into the following eight categories: (1) functional status, which refers to measures of (I) ADL; (2) mobility status, which refers to measures of motor performance or PA behavior; (3) hospital outcomes, which refers to measures of healthcare utilization during hospitalization (e.g., length of stay [LOS], hospital costs]; (4) adverse clinical events, which refer to measures of falls, medical complications, or mortality; (5) psychological status, which refers to measures of health-related quality of life (HRQOL), 
Table 2 Characteristics of the included studies

\begin{tabular}{|c|c|c|c|c|c|}
\hline $\begin{array}{l}\text { Study } \\
\text { Country }\end{array}$ & Sample & Intervention & $\begin{array}{l}\text { Outcome measures during } \\
\text { hospitalization* } \\
\text { (category: outcome measure) } \\
\text { *primary outcome measure in } \\
\text { bold }\end{array}$ & $\begin{array}{l}\text { Time point of } \\
\text { measurement } \\
\text { (primary } \\
\text { outcome) }\end{array}$ & Main findings \\
\hline $\begin{array}{l}\text { Abizanda } \\
2011 \text { [32] } \\
\text { Spain }\end{array}$ & $\begin{array}{l}n=400 \\
\text { Mean age, } 84 \text { yrs. } \\
\text { Females: } n=227 \\
(57 \%) ; \\
\text { Patients with acute } \\
\text { medical illness } \\
\text { (stroke, } \\
\text { cardiopulmonary } \\
\text { pathologies, or } \\
\text { other diagnoses) }\end{array}$ & $\begin{array}{l}\text { Intervention: } \\
\text { - Additional occupational } \\
\text { therapy by special trained } \\
\text { therapists (daily } 45 \text {-min ses- } \\
\text { sions, } 5 \text { days/week) } \\
\text { - Day 1: physical, functional, } \\
\text { cognitive social and } \\
\text { emotional assessment; } \\
\text { preparation of individual } \\
\text { therapeutic plan } \\
\text { - Day } 2 \text { until discharge: } \\
\text { cognitive exercises, ADL } \\
\text { training (mobility in bed, } \\
\text { sitting and standing, chair to } \\
\text { bed transfers, wheelchair to } \\
\text { bed/toilet transfers, dressing, } \\
\text { bathing, personal hygiene, } \\
\text { toilet use) }\end{array}$ & $\begin{array}{l}\text { FCT: Barthel Index } \\
\text { (improvement of } \geq 10 \text { pt. from } \\
\text { admission to discharge) } \\
\text { COG: CAM }\end{array}$ & $\begin{array}{l}\text { Admission } \\
\text { Discharge }\end{array}$ & $\begin{array}{l}\text { Between-group differences at } \\
\text { discharge: } \\
\text { - Improvement in Barthel } \\
\text { Index of } \geq 10 \text { pt. from } \\
\text { admission to discharge: n.s. } \\
\text { (total sample, stroke/ } \\
\text { cardiopulmonary patients), } \uparrow \\
\text { (others) } \\
\text { - Absolute improvement in } \\
\text { Barthel Index: n.s. (total } \\
\text { sample, stroke patients, } \\
\text { others), } \uparrow \text { (cardiopulmonary } \\
\text { patients) } \\
\text { Feasibility: } \\
\text { - Missing data: Barthel } \\
\text { Index =0\% (admission), } 6 \% \\
\text { (discharge) }\end{array}$ \\
\hline
\end{tabular}

MOB: Handgrip strength (handheld dynamometry)

FCT: Katz ADL Index

BPN: Body weight, energy intake, protein intake, calf and arm circumferences, triceps skin fold, biochemical measures (serum albumin, C-reactive protein)
Brown $2016 n=100$

[34]

USA
Mean age: $74 \mathrm{yrs}$. Females: $n=3(3 \%)$ Patients with acute medical illness
- Day of discharge: a second

30-min session in addition to the regular 45-min daily intertives or caregivers; recommendations for $\mathrm{ADL}$ at

Control: Conventional physiotherapy

Intervention: physiotherapy (start: day 1 or 2, 2 times/day for 30 min, 5 days/week),

- Focus on leg extension exercises in the upright position

- Nutritional supplements

Control:

- Walking with/without technical assistance or human help (start: day 3 to 6,3 times/week until discharge)

- Nutritional supplements

- Physical therapy at home for 1 month

Intervention:

- Additional mobility protocol: Starting with basic transfers with progress to ambulation if tolerated (2 times/day, 1520 min, 7 days/week)

- Patients were encouraged to walk at each session

- Physical activity behavioral strategy: goal setting, diary and interview to increase times out of bed Control: Usual care (physical therapy had to be ordered by physicians)

\section{FCT: Modified Katz ADL} Index

HU: LOS, physical therapy ordered during hospitalization ACE: Falls

\section{Admission Clinical stable condition}

Admission Discharge

Between-group differences at discharge:

- Modified Katz ADL Index:

n.s.

Changes during

hospitalization in total

sample:

- Katz ADL Index: n.s.

Group $\times$ time interaction during hospitalization:

- Katz ADL Index: n.s.

Feasibility:

- Katz ADL Index: mean admission score in both groups was within the best $15 \%$ of the rating scale $\rightarrow$ ceiling effect 
Table 2 Characteristics of the included studies (Continued)

\begin{tabular}{|c|c|c|c|c|c|}
\hline $\begin{array}{l}\text { Study } \\
\text { Country }\end{array}$ & Sample & Intervention & $\begin{array}{l}\text { Outcome measures during } \\
\text { hospitalization* } \\
\text { (category: outcome measure) } \\
\text { *primary outcome measure in } \\
\text { bold }\end{array}$ & $\begin{array}{l}\text { Time point of } \\
\text { measurement } \\
\text { (primary } \\
\text { outcome) }\end{array}$ & Main findings \\
\hline $\begin{array}{l}2013 \text { [35] } \\
\text { Poland }\end{array}$ & $\begin{array}{l}\text { Mean age: } 76 \text { yrs. } \\
\text { Females: } n=14 \\
(41 \%) ; \\
\text { Patients with major } \\
\text { abdominal surgery }\end{array}$ & $\begin{array}{l}\text { - Usual care with a modified } \\
\text { exercise component based } \\
\text { on the Proprioceptive } \\
\text { Neuromuscular Facilitation } \\
\text { concept ( } 30 \text { min/day) } \\
\text { Control: Usual care ( } 30 \mathrm{~min} / \\
\text { day) }\end{array}$ & $\begin{array}{l}\text { FCT: Lawton IADL scale } \\
\text { MOB: UCLA scale, PPSA } \\
\text { BPN: Forced ventilation } \\
\text { capacity, first-second forced ex- } \\
\text { piratory volume, maximal ex- } \\
\text { piratory flow (spirometry) } \\
\text { HU: LOS }\end{array}$ & $\begin{array}{l}\text { surgery } \\
4 \text { days after } \\
\text { surgery }\end{array}$ & $\begin{array}{l}\text { days prior surgery to } 4 \text { days } \\
\text { after surgery: } \\
\text { - 10MWT, TUG: } \downarrow \text { in both } \\
\text { groups } \\
\text { - Lawton IADL scale, UCLA, } \\
\text { PPSA: NA } \\
\text { Between-group differences } 4 \\
\text { days after surgery: } \\
\text { - PPSA: } \uparrow \\
\text { - 10MWT, TUG: NA } \\
\text { Feasibility: } \\
\text { - Lawton IADL scale: mean } \\
\text { admission score of the } \\
\text { sample was within the best } \\
\text { 15\% of the rating scale } \rightarrow \\
\text { ceiling effect } \\
\text { - Missing data (3 days prior \& } \\
4 \text { days after surgery): } \\
\text { 10MWT, TUG = 9\%, SAP = } \\
\text { 0\%, UCLA, IADL: NA }\end{array}$ \\
\hline $\begin{array}{l}\text { Eyres } 2005 \\
\text { [36] } \\
\text { Australia }\end{array}$ & $\begin{array}{l}n=15 \\
\text { Mean age: } 80 \text { yrs. } \\
\text { Females: } n=9 \\
(60 \%) ; \\
\text { Patients with acute } \\
\text { medical illness }\end{array}$ & $\begin{array}{l}\text { Intervention: } \\
\text { - Daily additional occupational } \\
\text { therapy } \\
\text { - Self-care program (ADL), IADL } \\
\text { training (e.g., cooking, laun- } \\
\text { dry, café visits), community } \\
\text { mobility (e.g., walking } \\
\text { outdoors) } \\
\text { Control: Usual care }\end{array}$ & $\begin{array}{l}\text { FCT: FIM } \\
\text { PSY: Self-Efficacy Gauge, Life } \\
\text { Satisfaction Index } \\
\text { HU: LOS, use of allied health } \\
\text { services, use of community } \\
\text { services, discharge destination }\end{array}$ & $\begin{array}{l}\text { Admission } \\
\text { Discharge }\end{array}$ & $\begin{array}{l}\text { Within-group comparisons } \\
\text { over time: } \\
\text { - FIM } \uparrow(I G, C G) \\
\text { - Self-Efficacy Gauge: n.s. (IG, } \\
\text { CG) } \\
\text { - Life Satisfaction Index: n.s. } \\
\text { (IG, CG) } \\
\text { Feasibility: } \\
\text { - Missing data: FIM, Self- } \\
\text { Efficacy Gauge, Life Satisfac- } \\
\text { tion Index =0\% }\end{array}$ \\
\hline $\begin{array}{l}\text { Hagsten } \\
2004[37] \\
\text { Sweden }\end{array}$ & $\begin{array}{l}n=100 \\
\text { Mean age: } 80 \text { yrs. } \\
\text { Females: } n=80 \\
(80 \%) ; \\
\text { Patients with hip } \\
\text { fracture }\end{array}$ & $\begin{array}{l}\text { Intervention: } \\
\text { - Additional occupational } \\
\text { therapy (40-60 min, } 5 \text { days/ } \\
\text { week) } \\
\text { - Self-care, independence at } \\
\text { home (transfers, bathroom } \\
\text { visits, morning activities, } \\
\text { dressing), use of aids } \\
\text { - Home visits } \\
\text { - Instruction of a }\end{array}$ & $\begin{array}{l}\text { FCT: Modified Klein-Bell ADL } \\
\text { Scale ( } 75 \text { items of } 4 \text { areas: } \\
\text { dressing, toilet visits, mobil- } \\
\text { ity, bathing/hygiene); mDRI } \\
\text { with visual analogize scales } \\
\text { for ADL, indoor IADL, and } \\
\text { outdoor IADL } \\
\text { PSY: Study-specific mDRI items } \\
\text { on fear of performing (I)ADL } \\
\text { and for pain level during (I)ADL }\end{array}$ & Discharge & $\begin{array}{l}\text { Between-group differences at } \\
\text { discharge: } \\
\text { - Modified Klein-Bell ADL } \\
\text { scale: dressing } \uparrow \text {, toilet visits } \\
\uparrow, \text { hygiene } \uparrow, \text { mobility: } n . s . \\
\text { mDRI: ADL, indoor/outdoor } \\
\text { IADLs, fear, pain: n.s. } \\
\text { Feasibility: } \\
\text { - Missing data: Klein-Bell ADL } \\
\text { scale, mDRI = 0\% }\end{array}$ \\
\hline
\end{tabular}

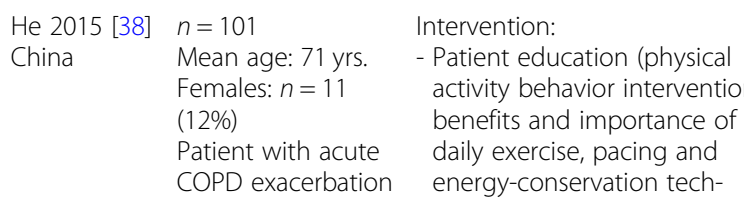

(category: outcome measure) * primary outcome measure in

FCT: Lawton IADL scale UCLA scale, PPSA $\mathrm{HU}:$ LOS

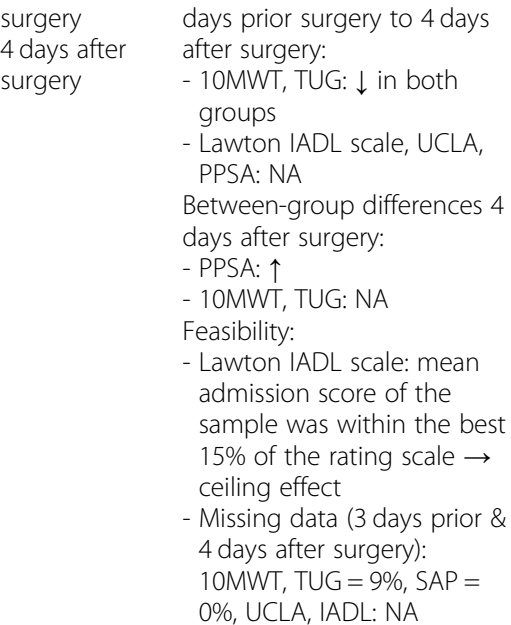

Between-group differences at

scale: dressing $\uparrow$, toilet visits $\uparrow$, hygiene $\uparrow$, mobility: n.s. ADLs, fear, pain: n.s.

scale, $\mathrm{mDRl}=0 \%$

\section{MOB: 6MWT}

DS: mMRC dyspnea grade,

\section{ADL-Dyspnea scale, CRQ-}

SAS, CAT Borg dyspnea scale, Bode index

BPN: Resting/exercise oxygen saturation (spirometry, arterial blood gas analysis)
Admission

Discharge
Within-group differences from admission to discharge: - 6MWT: $\uparrow(I G)$, n.s. (CG) - mMRC dyspnea grade: $\uparrow$ (IG), n.s. (CG)

- ADL-Dyspnea scale: $\uparrow(\mathrm{IG})$, n.s. (CG)

- CRQ-SAS: $\uparrow$ (IG), n.s. (CG)

- CAT: $\uparrow$ in both groups Feasibility: NA 
Table 2 Characteristics of the included studies (Continued)

\begin{tabular}{|c|c|c|c|c|c|}
\hline $\begin{array}{l}\text { Study } \\
\text { Country }\end{array}$ & Sample & Intervention & $\begin{array}{l}\text { Outcome measures during } \\
\text { hospitalization* } \\
\text { (category: outcome measure) } \\
\text { *primary outcome measure in } \\
\text { bold }\end{array}$ & $\begin{array}{l}\text { Time point of } \\
\text { measurement } \\
\text { (primary } \\
\text { outcome) }\end{array}$ & Main findings \\
\hline & & $\begin{array}{l}\text { exercise } \\
\text { - } 30 \text { min } 2 \text { times/day } \\
\text { Control: Usual care }\end{array}$ & & & \\
\hline $\begin{array}{l}\text { Jeffs } 2013 \\
{[39]} \\
\text { Australia }\end{array}$ & $\begin{array}{l}n=649 \\
\text { Mean age: } 79 \text { yrs. } \\
\text { Females: } n=340 \\
(52 \%) \\
\text { Patients with acute } \\
\text { medical illness }\end{array}$ & $\begin{array}{l}\text { Intervention: } \\
\text { - Graded physical activity and } \\
\text { orientation program twice } \\
\text { daily in addition to usual care } \\
\text { - Physical activity program: } \\
\text { progressive, variable } \\
\text { resistance training against } \\
\text { gravity, body or light weight } \\
\text { (progression whenever a } \\
\text { patient could perform } 10 \\
\text { repetitions), } \\
\text { - Cognitive exercise program: } \\
\text { Orientation, (7 questions for } \\
\text { improving orientation [day, } \\
\text { month, year, date, ward, bed } \\
\text { number, name of primary } \\
\text { nurse]); } \\
\text { - } 2 \text { times/day, } 5 \text { days/week, 20- } \\
30 \text { min until discharge + self- } \\
\text { training on weekends } \\
\text { Control: Usual care (including: } \\
24 \mathrm{~h} \text { nursing care, daily medical } \\
\text { assessment, allied health } \\
\text { referral) }\end{array}$ & $\begin{array}{l}\text { COG: Number of delirious } \\
\text { patients, severity/duration of } \\
\text { delirium (CAM) } \\
\text { HU: Discharge destination, LOS }\end{array}$ & $\begin{array}{l}\text { Admission } \\
\text { Every } 48 \mathrm{~h} \\
\text { until } \\
\text { discharge }\end{array}$ & $\begin{array}{l}\text { Between-group differences } \\
\text { - Number of delirious } \\
\text { patients: n.s. } \\
\text { Feasibility: } \\
\text { - No delirium in } 94 \% \text { of } \\
\text { patients } \rightarrow \text { rare event }\end{array}$ \\
\hline $\begin{array}{l}\text { Jones } 2006 \\
\text { [40] } \\
\text { Australia }\end{array}$ & $\begin{array}{l}n=160 \\
\text { Mean age: } 82 \text { yrs. } \\
\text { Females: } n=92 \\
(58 \%) \\
\text { Patients with acute } \\
\text { medical illness }\end{array}$ & $\begin{array}{l}\text { Intervention: } \\
\text { - Additional exercise program } \\
\text { (2 times/day, } 30 \text { min) } \\
\text { - Strengthening and mobility } \\
\text { exercises (e.g., sit-to-stand } \\
\text { transfer) specifically designed } \\
\text { to be carried out in a hospital } \\
\text { setting } \\
\text { Control: Usual care with } \\
\text { standard physiotherapy }\end{array}$ & $\begin{array}{l}\text { FCT: Barthel Index } \\
\text { MOB: TUG } \\
\text { HU: Discharge destination, LOS } \\
\text { ACE: Falls, mortality, } \\
\text { deterioration in medical status }\end{array}$ & $\begin{array}{l}\text { Admission } \\
\text { Discharge }\end{array}$ & $\begin{array}{l}\text { Between-group differences at } \\
\text { discharge: } \\
\text { - Barthel Index: n.s. } \\
\text { Multivariable regression } \\
\text { analyses: } \\
\text { - Barthel Index: Iow admission } \\
\text { Barthel Index \& IG } \\
\text { assignment = independent } \\
\text { predictors of improving } \\
\text { Barthel Index } \\
\text { Feasibility: } \\
\text { - Missing data: Barthel } \\
\text { Index }=0 \%\end{array}$ \\
\hline $\begin{array}{l}\text { Kimmel } 2016 \\
\text { [41] } \\
\text { Australia }\end{array}$ & $\begin{array}{l}n=92 \\
\text { Mean age: } 81 \text { yrs. } \\
\text { Females } n=59 \\
(64 \%) \\
\text { Patients with hip } \\
\text { fracture }\end{array}$ & $\begin{array}{l}\text { Intervention: } \\
\text { - Two additional physiotherapy } \\
\text { sessions aimed to improve } \\
\text { the functional advances } \\
\text { achieved during the usual } \\
\text { physiotherapy session (3 } \\
\text { times/day, } 7 \text { days/week) } \\
\text { Control: Usual care } \\
\text { (physiotherapy: } 1 \text { time/day, } 7 \\
\text { days/week) }\end{array}$ & $\begin{array}{l}\text { MOB: mILOAS, TUG } \\
\text { HU: LOS, Discharge destination, } \\
\text { opioid equivalence score } \\
\text { ACE: Postoperative } \\
\text { complications } \\
\text { PSY: Self-developed pain scale }\end{array}$ & Day 5 & $\begin{array}{l}\text { Between-group differences at } \\
\text { post-operative Day 5: } \\
\text { - mILOAS: n.s. } \\
\text { Between-group differences } \\
\text { controlled for confounding } \\
\text { factors: } \\
\text { - mILOAS: } \uparrow \\
\text { Feasibility: } \\
\text { - Missing data: } \text { mILOA }=0 \%\end{array}$ \\
\hline $\begin{array}{l}\text { Nikolaus } \\
1999[42] \\
\text { Germany }\end{array}$ & $\begin{array}{l}n=545 \\
\text { Mean age, } 81 \text { yrs. } \\
\text { Females: } n=400 \\
\text { (73\%); } \\
\text { Patients with acute } \\
\text { medical illness }\end{array}$ & $\begin{array}{l}\text { Intervention 1:Comprehensive } \\
\text { geriatric assessment and } \\
\text { interdisciplinary intervention in } \\
\text { the hospital and at home, } \\
\text { physical and occupational } \\
\text { therapy (washing, eating, } \\
\text { dressing, walking) twice a week } \\
\text { up to twice a day for } 30 \text { min } \\
\text { Intervention 2: Comprehensive } \\
\text { geriatric assessment with } \\
\text { recommendation in the } \\
\text { hospital and usual care at }\end{array}$ & $\begin{array}{l}\text { FCT: Barthel Index, Lawton } \\
\text { IADL scale } \\
\text { HU: Discharge destination, LOS }\end{array}$ & $\begin{array}{l}\text { Admission } \\
\text { Discharge }\end{array}$ & $\begin{array}{l}\text { Between-group differences at } \\
\text { discharge: } \\
\text { - Barthel Index, Lawton IADL } \\
\text { scale: n.s. } \\
\text { Feasibility: } \\
\text { - Barthel Index, Lawton IADL } \\
\text { scale: mean discharge } \\
\text { scores in both groups } \\
\text { within the best } 15 \% \text { of the } \\
\text { rating scale } \rightarrow \text { ceiling effect } \\
\text { - Missing data: Barthel Index, } \\
\text { Lawton IADL scale }=0 \%\end{array}$ \\
\hline
\end{tabular}


Table 2 Characteristics of the included studies (Continued)

\begin{tabular}{|c|c|c|c|c|}
\hline $\begin{array}{l}\text { Study } \\
\text { Country }\end{array}$ & Sample & Intervention & $\begin{array}{l}\text { Outcome measures during } \\
\text { hospitalization* } \\
\text { (category: outcome measure) } \\
\text { *primary outcome measure in } \\
\text { bold }\end{array}$ & $\begin{array}{l}\text { Time point of } \\
\text { measurement } \\
\text { (primary } \\
\text { outcome) }\end{array}$ \\
\hline
\end{tabular}

\begin{tabular}{|c|c|c|}
\hline & & $\begin{array}{l}\text { home } \\
\text { Control: Assessment of ADL } \\
\text { and cognition and usual care } \\
\text { in the hospital and at home }\end{array}$ \\
\hline $\begin{array}{l}\text { Oldmeadow } \\
2006 \text { [43] } \\
\text { Australia }\end{array}$ & $\begin{array}{l}n=60 \\
\text { Mean age: } 79 \text { yrs. } \\
\text { Females: } n=43 \\
(68 \%) \\
\text { Patient with hip } \\
\text { fracture }\end{array}$ & $\begin{array}{l}\text { Intervention: } \\
\text { - First walk at day } 1 \text { or } 2 \text { (early } \\
\text { mobilization) (7 days/week) } \\
\text { Control: Usual care (first walk at } \\
\text { day } 3 \text { or 4) (7 days/week) }\end{array}$ \\
\hline
\end{tabular}

MOB: mILOAS items: Transfer Day 7

from supine to sitting,

transfer from sitting to

standing (independent vs.

assisted), ambulation

(walking distance), step

negotiation (independent vs.

failed/unable)

$\mathrm{HU}$ : Discharge destination, LOS

\begin{tabular}{|c|c|c|}
\hline $\begin{array}{l}\text { Siebens et } \\
\text { al., } 2000 \text { [44] } \\
\text { USA }\end{array}$ & $\begin{array}{l}n=300 \\
\text { Mean age: } 78 \text { yrs. } \\
\text { Females: } n=182 \\
(61 \%) ; \\
\text { Patients with acute } \\
\text { medical illness }\end{array}$ & $\begin{array}{l}\text { Intervention: } \\
\text { - Hospital-based exercise pro- } \\
\text { gram (twice a day) } \\
\text { - Flexibility and strengthening } \\
\text { exercises } \\
\text { - Walking program ( } 60 \text { to } 80 \% \\
\text { max. Heart rate, } 5 \text { min to } 30 \\
\text { min) } \\
\text { Control: Usual care }\end{array}$ \\
\hline $\begin{array}{l}\text { Torres- } \\
\text { Sanchez } \\
2017 \text { [45] } \\
\text { Spain }\end{array}$ & $\begin{array}{l}n=58 \\
\text { Mean age: } 74 \text { yrs. } \\
\text { Females: } n=16 \\
(28 \%) ; \\
\text { Patients with acute } \\
\text { exacerbation of } \\
\text { COPD }\end{array}$ & $\begin{array}{l}\text { Intervention: } \\
\text { - Additional individually- } \\
\text { adapted endurance training } \\
\text { on a pedal exerciser } \\
\text { - Cycling time, velocity, and } \\
\text { resistance were adapted to } \\
\text { patient and increased every } \\
\text { day } \\
\text { Control: Usual care (no } \\
\text { supervised or progressive } \\
\text { exercise) }\end{array}$ \\
\hline
\end{tabular}

Asplund

$n=444$

Mean age: 81 yrs.

2000 [46]

Females: $n=251$

(61\%)

Patients with acute medical illness

Intervention:

- Multidisciplinary teamwork

(internist, geriatrician, nurses, nurse aids, physiotherapist,

occupational therapist, social worker, dietician)

- Assessment by

physiotherapist and

occupational therapist

- Early start of rehabilitation

- Discharge planning

Control: General medical unit care

$\begin{array}{ll}\text { Barnes } 2012 & n=1632 \\ \text { [47] } & \text { Mean age: } 81 \text { yrs. } \\ \text { USA } & \text { Females: } n=1094 \\ & (67 \%) \\ & \text { Patient with acute } \\ & \text { medical illness }\end{array}$

Intervention:

- Prepared environment (e.g., carpeting, handrails, uncluttered hallways)

- Patient-centered care (daily assessment by nurse of physical, cognitive and psychosocial function

- Protocols to improve of ADL

MOB: Lower limb strength (handheld dynamometer), balance (OLS), exercise capacity (30CST), physical activity/number of steps (SenseWear Armband)

\section{ACE: Mortality}

HU: LOS, discharge destination, Discharge
HU: LOS, hospital costs, process-of-care measures (physical therapy consults, orders for bed rest, use of physical restraints, documentation of discharge planning, discharge destination)

FCT: Katz ADL Index (bathing, dressing, toileting, transferring,

\author{
HU: LOS \\ ACE: Mortality \\ Discharge \\ Between-group differences at \\ discharge: \\ - LOS: n.s. \\ Feasibility: \\ - Missing data: $\operatorname{LOS}=0 \%$
}

(discharge)

Between-group differences at post-surgery day 7 :

- mILOAS: transfer item: $\uparrow$. walking distance: $\uparrow$, step negotiation: n.s.

Feasibility at day 7 :

- mILOAS step negotiation item: $>15 \%(23 \%)$ of total sample with worst possible score $\rightarrow$ floor effect, $21 \%$ missing data

- mILOAS transfer item $=15 \%$ missing data

Admission

Admission

Discharge

Group $\times$ time interaction:

- Lower-limb strength: $\uparrow$

- Balance: $\uparrow$

- Exercise capacity: $\uparrow$

Between-group differences at discharge:

- Lower-limb strength: $\uparrow$

- Balance (OLS): $\uparrow$

- Exercise capacity (30STS): n.s. Feasibility:

- Missing data: Lower-limb strength, balance (OLS), exercise capacity $(30 \mathrm{CST})=0 \%$ Between-group differences at discharge:

- Mortality: n.s.

Feasibility:

- Missing data: mortality = 3\%

$97 \%$ survivals $\rightarrow$ mortality $=$ rare event

Admission

Discharge

Between-group differences at discharge:

- LOS: $\downarrow$

- Hospital costs: $\downarrow$

- Feasibility:

- Missing data: LOS, hospital costs $=$ NA 
Table 2 Characteristics of the included studies (Continued)

\begin{tabular}{|c|c|c|c|c|c|}
\hline $\begin{array}{l}\text { Study } \\
\text { Country }\end{array}$ & Sample & Intervention & $\begin{array}{l}\text { Outcome measures during } \\
\text { hospitalization* } \\
\text { (category: outcome measure) } \\
\text { *primary outcome measure in } \\
\text { bold }\end{array}$ & $\begin{array}{l}\text { Time point of } \\
\text { measurement } \\
\text { (primary } \\
\text { outcome) }\end{array}$ & Main findings \\
\hline & & $\begin{array}{l}\text { (bathing/dressing, mobility/ } \\
\text { transferring, toileting, } \\
\text { feeding), nutrition, skin care, } \\
\text { falls, cognition, mood etc., } \\
\text { daily team rounds by } \\
\text { physiotherapist, nurse, social } \\
\text { worker, nutritionist) } \\
\text { - Planning for discharge } \\
\text { - Medical care review (daily by } \\
\text { medical director) } \\
\text { - Protocols to minimize adverse } \\
\text { effects (e.g., urinary } \\
\text { catheterization) } \\
\text { Control: Usual care }\end{array}$ & $\begin{array}{l}\text { eating), Lawton IADL scale } \\
\text { (shopping, cooking, performing } \\
\text { household chores, using } \\
\text { transportation, managing } \\
\text { money, managing medication, } \\
\text { and using the telephone) } \\
\text { MOB: 5-items hierarchical mo- } \\
\text { bility scale } \\
\text { ACE: Mortality }\end{array}$ & & \\
\hline $\begin{array}{l}\text { Counsell } \\
2000 \text { [48] } \\
\text { USA }\end{array}$ & $\begin{array}{l}n=1531 \\
\text { Mean age: } 80 \text { yrs. } \\
\text { Females: } n=926 \\
(61 \%) \\
\text { Patients with acute } \\
\text { medical illness }\end{array}$ & $\begin{array}{l}\text { Intervention: } \\
\text { - Prepared environment (e.g., } \\
\text { carpeting, handrails, } \\
\text { uncluttered hallways) } \\
\text { - Patient-centered care (daily } \\
\text { assessment by nurse of phys- } \\
\text { ical, cognitive and psycho- } \\
\text { social function } \\
\text { - Protocols to improve of ADL } \\
\text { (bathing/dressing, mobility/ } \\
\text { transferring, toileting, feeding) } \\
\text { nutrition, skin care, falls, } \\
\text { cognition, mood etc., daily } \\
\text { team rounds by } \\
\text { physiotherapist, nurse, social } \\
\text { worker, nutritionist) } \\
\text { - Planning for discharge } \\
\text { - Medical care review (daily by } \\
\text { medical director) } \\
\text { - Protocols to minimize adverse } \\
\text { effects } \\
\text { Control: Usual care }\end{array}$ & $\begin{array}{l}\text { FCT: Modified Katz ADL } \\
\text { Index (bathing, dressing, } \\
\text { toileting, transferring, eating), } \\
\text { modified Lawton IADL scale } \\
\text { (shopping, cooking, performing } \\
\text { household chores, using } \\
\text { transportation, managing } \\
\text { money, managing medication, } \\
\text { and using the telephone) } \\
\text { MOB: PPME, 5-items hierarch- } \\
\text { ical mobility scale } \\
\text { HU: Process-of-care measures } \\
\text { (nursing care plans, time from } \\
\text { admission to initiation of dis- } \\
\text { charge planning, social work } \\
\text { consultation, orders for bed } \\
\text { rest, physical therapy consults, } \\
\text { use of urinary catheters, and } \\
\text { application of physical re- } \\
\text { straints, inappropriate medica- } \\
\text { tions), LOS, hospital costs, } \\
\text { discharge destination } \\
\text { PSY: Caregiver satisfaction } \\
\text { ACE: Mortality }\end{array}$ & $\begin{array}{l}\text { Admission } \\
\text { Discharge }\end{array}$ & $\begin{array}{l}\text { Between-group differences at } \\
\text { discharge: } \\
\text { - Mortality: n.s.; Modified Katz } \\
\text { ADL Index: n.s. } \\
\text { Feasibility: } \\
\text { - Missing data: Katz ADL } \\
\text { Index = NA (admission \& } \\
\text { discharge) }\end{array}$ \\
\hline $\begin{array}{l}\text { Huusko } 2000 \\
\text { [49] } \\
\text { Finland }\end{array}$ & $\begin{array}{l}n=260 \\
\text { Mean age: } 80 \text { yrs. } \\
\text { Females: } n=184 \\
(72 \%) \\
\text { Patients with hip } \\
\text { fracture } \\
\text { No dementia } \\
\text { (MMSE 24-30): } n= \\
\text { 99 (41\%) } \\
\text { Suspected severe } \\
\text { dementia (MMSE } \\
0-11): n=28 \text { (12\%) } \\
\text { Suspected } \\
\text { moderate } \\
\text { dementia (MMSE } \\
\text { 12-17): } n=36 \\
(15 \%) \\
\text { Suspected mild } \\
\text { dementia (MMSE } \\
\text { 18-23): } n=77 \\
(32 \%)\end{array}$ & $\begin{array}{l}\text { Intervention: } \\
\text { - Multidisciplinary teamwork } \\
\text { (geriatrician, general } \\
\text { practitioner, nurses, social } \\
\text { worker, neuropsychologist, } \\
\text { occupational therapist, } \\
\text { physiotherapist) } \\
\text { - Geriatric team assessment } \\
\text { - Physiotherapy (2times/day), } \\
\text { ADL training by nurses } \\
\text { - Weekly meetings by } \\
\text { physiotherapists and nurses } \\
\text { - Discharge plan } \\
\text { Control: Discharged to local } \\
\text { hospitals }\end{array}$ & HU: LOS & Discharge & $\begin{array}{l}\text { Between-group differences at } \\
\text { discharge: } \\
\text { - LOS: severe dementia } \\
\text { (MMSE score: } 0 \text { - } 11 \text { pt): n.s.; } \\
\text { moderate dementia (MMSE } \\
\text { score: } 12-17 \text { pt): } \downarrow \text {; mild } \\
\text { dementia (MMSE score: } 18- \\
23 \text { pt) } \downarrow \text {; normal (MMSE } \\
\text { score: } 24-30 \text { ): } \text { n.s. } \\
\text { Feasibility: } \\
\text { - Missing data: } \text { LOS }=0 \%\end{array}$ \\
\hline $\begin{array}{l}\text { Landefeld } \\
1995[50] \\
\text { USA }\end{array}$ & $\begin{array}{l}n=651 \\
\text { Mean age: } 80 \text { yrs. } \\
\text { Females: } n=435 \\
(67 \%)\end{array}$ & $\begin{array}{l}\text { Intervention: } \\
\text { - Prepared environment (e.g., } \\
\text { carpeting, handrails, } \\
\text { uncluttered hallways) }\end{array}$ & $\begin{array}{l}\text { FCT: Modified Katz ADL } \\
\text { Index (bathing, dressing, } \\
\text { toileting, transferring, eating), } \\
\text { Lawton IADL scale }\end{array}$ & $\begin{array}{l}\text { Admission } \\
\text { Discharge }\end{array}$ & $\begin{array}{l}\text { Between-group differences at } \\
\text { discharge: } \\
\text { - Katz ADL Index: } \uparrow \\
\text { Multivariable regression }\end{array}$ \\
\hline
\end{tabular}


Table 2 Characteristics of the included studies (Continued)

\begin{tabular}{|c|c|c|c|c|c|}
\hline $\begin{array}{l}\text { Study } \\
\text { Country }\end{array}$ & Sample & Intervention & $\begin{array}{l}\text { Outcome measures during } \\
\text { hospitalization* } \\
\text { (category: outcome measure) } \\
\text { *primary outcome measure in } \\
\text { bold }\end{array}$ & $\begin{array}{l}\text { Time point of } \\
\text { measurement } \\
\text { (primary } \\
\text { outcome) }\end{array}$ & Main findings \\
\hline & $\begin{array}{l}\text { Patients with acute } \\
\text { medical illness }\end{array}$ & $\begin{array}{l}\text { - Patient-centered care (daily } \\
\text { assessment by nurse of phys- } \\
\text { ical, cognitive and psycho- } \\
\text { social function } \\
\text { - Protocols to improve of ADL } \\
\text { (bathing/dressing, mobility/ } \\
\text { transferring, toileting, feeding) } \\
\text { nutrition, skin care, falls, } \\
\text { cognition, mood etc., daily } \\
\text { team rounds by } \\
\text { physiotherapist, nurse, social } \\
\text { worker, nutritionist) } \\
\text { - Planning for discharge } \\
\text { - Medical care review (daily by } \\
\text { medical director) } \\
\text { - Protocols to minimize adverse } \\
\text { effects (e.g., urinary }\end{array}$ & $\begin{array}{l}\text { MOB: Walking ability } \\
\text { HU: Discharge destination, LOS, } \\
\text { hospital costs } \\
\text { PSY: GDS, overall health status } \\
\text { COG: MMSE }\end{array}$ & & $\begin{array}{l}\text { analyses controlled for } \\
\text { confounding baseline patient } \\
\text { characteristics: IG } \\
\text { assignment = significant } \\
\text { independent predictor of an } \\
\text { increase in the number of } \\
\text { independently performed } \\
\text { ADLs } \\
\text { Feasibility: } \\
\text { - Katz ADL Index: > 15\% of } \\
\text { participants reaching a score } \\
\text { within the best 15\% of the } \\
\text { instrument's rating scale } \rightarrow \\
\text { ceiling effect } \\
\text { - Missing data: Katz ADL } \\
\text { Index: } 0 \% \text { (admission \& } \\
\text { discharge) }\end{array}$ \\
\hline
\end{tabular}

\begin{tabular}{|c|c|c|c|c|c|}
\hline $\begin{array}{l}\text { Naglie } 2002 \\
\text { [51] } \\
\text { Canada }\end{array}$ & $\begin{array}{l}n=279 \\
\text { Mean age } 84 \text { yrs. } \\
\text { Females } n=223 \\
(80 \%) ; \\
\text { Patients with hip } \\
\text { fracture }\end{array}$ & $\begin{array}{l}\text { Intervention: } \\
\text { - Multidisciplinary teamwork } \\
\text { (physiotherapist, occupational } \\
\text { therapist, nurse, social worker) } \\
\text { - Special education of staff } \\
\text { - Prevention of complications } \\
\text { (e.g., delirium, urinary } \\
\text { problems, malnutrition) } \\
\text { - Physiotherapy: early full } \\
\text { weight bearing, ADL training, } \\
\text { (2 times/day for } 5 \text { day/week) } \\
\text { - Discharge plan, pre-discharge } \\
\text { home visits } \\
\text { - } 2 \text { times/week meeting for } \\
\text { monitoring treatment plan } \\
\text { Control: Usual care }\end{array}$ & $\begin{array}{l}\text { HU: Discharge destination, } \\
\text { LOS }\end{array}$ & $\begin{array}{l}\text { Admission } \\
\text { Discharge }\end{array}$ & $\begin{array}{l}\text { Between-group differences at } \\
\text { discharge: } \\
\text { - Discharge destination: } \uparrow \text { (in } \\
\text { community-dwellers, rela- } \\
\text { tive's/retirement home resi- } \\
\text { dents), n.s. (in nursing home } \\
\text { residents) } \\
\text { Feasibility: } \\
\text { - Missing data: Discharge } \\
\text { destination: } 0 \%\end{array}$ \\
\hline $\begin{array}{l}\text { Pitkälä } 2008 \\
\text { [52] } \\
\text { Finland }\end{array}$ & $\begin{array}{l}n=174 \\
\text { Mean age: } 83 \text { yrs. } \\
\text { Females: } n=128 \\
\text { (74\%) } \\
\text { Patients with } \\
\text { delirium }\end{array}$ & $\begin{array}{l}\text { Intervention: } \\
\text { - Comprehensive geriatric } \\
\text { assessment (physical } \\
\text { examination, cognition, } \\
\text { nutrition, screening of } \\
\text { depression, review of } \\
\text { medication) } \\
\text { - Administering antipsychotics } \\
\text { for hyperactive/psychotic } \\
\text { symptoms } \\
\text { - Cholinesterase inhibitors } \\
\text { - Orientation (calendars, clocks) } \\
\text { - Physiotherapy } \\
\text { - Nutritional supplements } \\
\text { - Comprehensive discharge } \\
\text { planning (e.g., occupational } \\
\text { home visits) } \\
\text { Control: Usual care }\end{array}$ & $\begin{array}{l}\text { PSY: 15D HRQOL } \\
\text { questionnaire, self- } \\
\text { developed subjective health } \\
\text { scale }\end{array}$ & $\begin{array}{l}\text { Admission } \\
\text { Discharge }\end{array}$ & $\begin{array}{l}\text { Between-group differences at } \\
\text { discharge: } \\
\text { - HRQOL: } \uparrow \\
\text { - Self-developed subjective } \\
\text { health sale: } \uparrow \\
\text { Feasibility: } \\
\text { - Missing data: 15D } \\
\text { questionnaire: 9\%; self- } \\
\text { developed subjective health } \\
\text { sale: NA (admission \& } \\
\text { discharge) }\end{array}$ \\
\hline $\begin{array}{l}\text { Prestmo } \\
2015 \text { [30] } \\
\text { Taraldsen } \\
2014 \text { [31] } \\
\text { Norway }\end{array}$ & $\begin{array}{l}n=397 \\
\text { Mean age: } 83 \text { yrs. } \\
\text { Females: } n=293 \\
\text { (73\%) } \\
\text { Patients with hip } \\
\text { fracture }\end{array}$ & $\begin{array}{l}\text { Intervention: } \\
\text { - Multidisciplinary teamwork } \\
\text { (geriatricians, nurses, } \\
\text { physiotherapists, occupational } \\
\text { therapists, with special } \\
\text { competence in geriatrics) } \\
\text { - Comprehensive geriatric } \\
\text { assessment (somatic and } \\
\text { mental health, function, social } \\
\text { situation) }\end{array}$ & $\begin{array}{l}\text { MOB: SPPB, PA (activPAL: } \\
\text { time spent in upright, } \\
\text { number of upright events), } \\
\text { Cumulated Ambulation Score } \\
\text { HU: LOS, discharge destination, } \\
\text { hospital costs }\end{array}$ & $\begin{array}{l}\text { Day } 4 \text { after } \\
\text { surgery } \\
\text { (activePAL) } \\
\text { Day } 5 \text { after } \\
\text { surgery (SPPB) }\end{array}$ & $\begin{array}{l}\text { Between-group differences at } \\
\text { day } 4 \text { (activePAL) and } 5 \\
\text { (SPPB): } \\
\text { - SPPB: } \uparrow \\
\text { - Time spent in upright: } \uparrow \\
\text { Feasibility: } \\
\text { - Missing data: SPPB }=13 \% \text { (5 } \\
\quad \text { days after surgery) } \\
\text { - activPAL: > 15\% missing } \\
\text { data }\end{array}$ \\
\hline
\end{tabular}


Table 2 Characteristics of the included studies (Continued)

\begin{tabular}{|c|c|c|c|c|c|}
\hline $\begin{array}{l}\text { Study } \\
\text { Country }\end{array}$ & Sample & Intervention & $\begin{array}{l}\text { Outcome measures during } \\
\text { hospitalization* } \\
\text { (category: outcome measure) } \\
\text { *primary outcome measure in } \\
\text { bold }\end{array}$ & $\begin{array}{l}\text { Time point of } \\
\text { measurement } \\
\text { (primary } \\
\text { outcome) }\end{array}$ & Main findings \\
\hline & & $\begin{array}{l}\text { - Interdisciplinary team } \\
\text { meetings } \\
\text { - Adequate nutrition, } \\
\text { - Individual rehabilitation plan } \\
\text { based on cognition and } \\
\text { motivation } \\
\text { - Early mobilization, functioning } \\
\text { in ADL, weight-bearing exer- } \\
\text { cise program } \\
\text { - Early discharge planning } \\
\text { Control: Usual care (standard } \\
\text { orthopedic care) }\end{array}$ & & & \\
\hline $\begin{array}{l}\text { Siebens et } \\
\text { al., } 2000 \text { [44] } \\
\text { USA }\end{array}$ & $\begin{array}{l}n=300 \\
\text { Mean age: } 78 \text { yrs. } \\
\text { Females: } n=182 \\
(61 \%) ; \\
\text { Patients with acute } \\
\text { medical illness }\end{array}$ & $\begin{array}{l}\text { Intervention: } \\
\text { - Hospital-based exercise pro- } \\
\text { gram (twice a day) } \\
\text { - Flexibility and strengthening } \\
\text { exercises } \\
\text { - Walking program ( } 60 \text { to } 80 \% \\
\text { max. Heart rate, } 5 \text { min to } 30 \\
\text { min) } \\
\text { Control: Usual care }\end{array}$ & $\begin{array}{l}\text { HU: LOS } \\
\text { ACE: Mortality }\end{array}$ & Discharge & $\begin{array}{l}\text { Between-group differences at } \\
\text { discharge: } \\
\text { - LOS: n.s. } \\
\text { Feasibility: } \\
\text { - Missing data: LOS }=0 \%\end{array}$ \\
\hline \multirow[t]{2}{*}{$\begin{array}{l}\text { Stenvall } \\
\text { 2007a,b, } \\
2012[27-29] \\
\text { Lundström } \\
\text { 2007 [26] } \\
\text { Sweden }\end{array}$} & $\begin{array}{l}\text { Total sample: } \\
n=199 \\
\text { Mean age: } 82 \text { yrs. } \\
\text { Females: } n=148 \\
\text { (74\%) } \\
\text { Patients with hip } \\
\text { fracture }\end{array}$ & $\begin{array}{l}\text { Intervention: } \\
\text { - Multidisciplinary teamwork } \\
\text { (nurses, physiotherapists, } \\
\text { occupational therapists, } \\
\text { dietician, geriatrician) } \\
\text { - Staff education in prevention } \\
\text { of postoperative complication } \\
\text { - Individual care planning (all } \\
\text { team members assessed each } \\
\text { patient as soon as possible, } \\
\text { planning of process and goals } \\
\text { twice a week) } \\
\text { - Prevention and treatment of } \\
\text { complications (falls, delirium } \\
\text { etc.) } \\
\text { - Pain treatment (contained } \\
\text { assessment of underlying } \\
\text { causes) } \\
\text { - Saturation (oxygen-enriched } \\
\text { air during first two } \\
\text { postoperative days) } \\
\text { - Nutrition (protein-enriched } \\
\text { meals during the first four } \\
\text { days) } \\
\text { - Mobilization: (ADL training } \\
\text { with focus on fall risk factors, }\end{array}$ & $\begin{array}{l}\text { ACE: Falls, fallers, and time } \\
\text { lapse to first fall after } \\
\text { admission; AIS, postoperative } \\
\text { complications (urinary tract } \\
\text { infections, decubitus ulcer, } \\
\text { sleeping disturbances, } \\
\text { mortality) } \\
\text { MOB: COVS walking item } \\
\text { FCT: ADL staircase (Katz ADL } \\
\text { Index with IADL items) } \\
\text { HU: Discharge destination, } \\
\text { LOS } \\
\text { COG: Number of delirious } \\
\text { days (OBS scale), MMSE } \\
\text { PSY: GDS } \\
\text { BPN: Nutritional problems } \\
\text { assessed by care/nursing staff } \\
\end{array}$ & Discharge & $\begin{array}{l}\text { Between-group differences at } \\
\text { discharge: } \\
\text { - Falls: } \downarrow \\
\text { - Fallers: } \downarrow \\
\text { - AlS: minor or moderate } \\
\text { injuries: } \downarrow \text {, serious injuries: n.s. } \\
\text { - COVS walking item: n.s. } \\
\text { - ADL staircase: NA (Katz ADL } \\
\text { Index: n.s., IADL: NA) } \\
\text { - Discharge destination: n.s. } \\
\text { - Number of delirious days: } \downarrow \\
\text { - MMSE: n.s. } \\
\text { - GDS: n.s. } \\
\text { Feasibility: } \\
\text { - Falls: } 81 \%=\text { non-fallers } \rightarrow \\
\text { rare event } \\
\text { - AIS: not assessable in } 81 \% \text {; } \\
\text { 42\% of fallers with an AIS } \\
\text { score of } 0 \text { pt. } \rightarrow \text { floor effect } \\
\text { - GDS: missing data at } \\
\text { discharge in } 20 \% \\
\text { - ADL staircase: }>15 \% \text { of } \\
\text { patients reaching a score } \\
\text { within the best } 15 \% \text { of the } \\
\text { best possible score } \rightarrow \\
\text { ceiling effect }\end{array}$ \\
\hline & $\begin{array}{l}\text { Subsample: } \\
n=64 \text { (32\%) } \\
\text { Mean age: } 82 \text { yrs. } \\
\text { Females: } n=47 \\
\text { (73\%) } \\
\text { Patients with hip } \\
\text { fracture \& } \\
\text { dementia } \\
\text { Mean MMSE score: } \\
8.6(I G), 6.9 \text { (CG) }\end{array}$ & $\begin{array}{l}\text { high-intensity weight-bearing } \\
\text { exercises) } \\
\text { Control: Usual care (no } \\
\text { corresponding team work) }\end{array}$ & $\begin{array}{l}\text { ACE: Postoperative } \\
\text { complications (pneumonia, } \\
\text { urinary tract infection, decubital } \\
\text { ulcers, new fracture, falls, fallers, } \\
\text { fall incidence rate, mortality) } \\
\text { COG: Number of delirious } \\
\text { days (OBS scale) } \\
\text { BPN: Nutritional problems } \\
\text { assessed by care/nursing staff } \\
\text { MOB: COVS walking item } \\
\text { FCT: ADL staircase (Katz ADL } \\
\text { Index with IADL items) }\end{array}$ & & $\begin{array}{l}\text { Between-group differences at } \\
\text { discharge: } \\
\text { - Postoperative complications: } \\
\text { total: NA; urinary tract } \\
\text { infection: } \downarrow \text {; fallers: } \downarrow \text {; Fall } \\
\text { incidence rate: } \downarrow \text {; mortality, } \\
\text { pneumonia, decubital ulcers, } \\
\text { new fracture: n.s. } \\
\text { - Number of delirious days: } \downarrow \\
\text { - COVS walking item: n.s. } \\
\text { - ADL staircase: NA (Katz ADL } \\
\text { Index: n.S., IADL: NA) }\end{array}$ \\
\hline $\begin{array}{l}\text { Vidan } 2005 \\
\text { [53] } \\
\text { Spain }\end{array}$ & $\begin{array}{l}n=319 \\
\text { Mean age: } 82 \text { yrs. } \\
\text { Females: } n=260\end{array}$ & $\begin{array}{l}\text { Intervention: } \\
\text { - Multidisciplinary teamwork } \\
\text { (geriatrician, rehabilitation }\end{array}$ & $\begin{array}{l}\text { HU: LOS } \\
\text { ACE: Mortality, postoperative } \\
\text { complications }\end{array}$ & $\begin{array}{l}\text { Admission } \\
\text { Discharge }\end{array}$ & $\begin{array}{l}\text { Admission to discharge: } \\
\text { - LOS: n.s. } \\
\text { - Mortality: } \downarrow\end{array}$ \\
\hline
\end{tabular}


Table 2 Characteristics of the included studies (Continued)

\begin{tabular}{|c|c|c|c|c|c|}
\hline $\begin{array}{l}\text { Study } \\
\text { Country }\end{array}$ & Sample & Intervention & $\begin{array}{l}\text { Outcome measures during } \\
\text { hospitalization* } \\
\text { (category: outcome measure) } \\
\text { *primary outcome measure in } \\
\text { bold }\end{array}$ & $\begin{array}{l}\text { Time point of } \\
\text { measurement } \\
\text { (primary } \\
\text { outcome) }\end{array}$ & Main findings \\
\hline & $\begin{array}{l}(82 \%) \\
\text { Patients with hip } \\
\text { fracture }\end{array}$ & $\begin{array}{l}\text { specialist, and specific social } \\
\text { worker) } \\
\text { - Geriatric assessment (medical, } \\
\text { psychosocial problems and } \\
\text { functional capability) } \\
\text { - Interdisciplinary meeting to } \\
\text { elaborate a comprehensive } \\
\text { therapeutic plan (weekly } \\
\text { repeated) } \\
\text { - Daily visits by geriatrician } \\
\text { - Rehabilitation specialist } \\
\text { planned physiotherapy } \\
\text { (schedule, intensity and } \\
\text { duration) } \\
\text { - Social worker assessed the } \\
\text { social environment } \\
\text { Control: Usual care }\end{array}$ & COG: CAM & & $\begin{array}{l}\text { - Postoperative complications: } \\
\downarrow \\
\text { Feasibility: } \\
\text { - LOS: } 0 \% \text { (admission to } \\
\text { discharge) } \\
\text { - Mortality: } 97 \% \text { survivals } \rightarrow \\
\text { rare event } \\
\text { - Postoperative complications: } \\
47 \% \text { of patients without } \\
\text { complications (admission to } \\
\text { discharge) } \rightarrow \text { rare events }\end{array}$ \\
\hline
\end{tabular}

10MWT 10-Meter Walking Test, 30CST 30-Seconds Chair Stand Test, 6MWT 6-Minute Walk Test, ACE Adverse clinical events, ADL Activities of daily living; AIS, Abbreviated Injury Scale, BPN Body constitution, physiological or nutritional status, CAM Confusion Assessment Method, CAM Confusion Assessment Method, CAT COPD Assessment Test, CG Control group, COG Cognitive functioning, COPD Chronic obstructive pulmonary disease, COVS Clinical Outcome Variables Scale, CRQSAS Chronic Respiratory Questionnaire Self-Administered Standardized, DSM-IV Diagnostic and Statistical Manual of Mental Disorders, FCT Functional status, FIM Functional Independence Measure, GDS Geriatric Depression Scale, HRQOL Health-related quality of life, HU Hospital outcomes, IADL Instrumental activities of daily living, IG Intervention group, LOS Length of stay, $m D R I$ modified Disability Rating Index, mILOAS Modified lowa level of Assistance, mMRC modified Medical Research Council, MMSE Mini-Mental State Examination, n.s not significant $(p>0.05)$, NA Not available, OLS One Leg Stance, PPAS Postoperative patient activity scale, PPME Physical Performance and Mobility Examination, PSY Psychological status, SPPB Short Physical Performance Battery, TUG Timed Up and Go, UCLA scale University of California, Los Angeles Activity scale; $\uparrow$, significant increase $(p \leq 0.05) ; \downarrow$, significant decrease $(p \leq 0.05)$

anxiety, depression, or confidence; (6) cognitive functioning, which refers to measures of global cognitive status or transient cognitive dysfunction (e.g., delirium); (7) body constitution, physiological or nutritional status, which refers to measures of lean and fat tissue mass, body weight, nutritional intake, or biochemical outcomes (e.g., serum albumin); and (8) disease-specific outcomes (e.g., COPD severity, exacerbation rates). In the following, the different primary outcome measures used across the included studies were described for each category. Due to their specificity, the disease-specific outcome measures were not further analyzed and discussed in this review.

\section{Functional status}

Functional status was assessed in 11 studies $(45.8 \%$; 8 exercise interventions [32-37, 40, 42] and 3 multidisciplinary programs $[28,48,50])$ using an (I) ADL measure only [32$36,40]$, both an ADL and IADL measure [37, 42, 48, 50], or a combined (I) ADL measure [28]. The most frequently used (I) ADL instruments were the Katz ADL Index [33, 34, 48, 50], the Barthel Index [32, 40, 42], and the Lawton IADL scale $[35,42]$. Other functional status measures included the Functional Independence Measure (FIM [36]), modified Disability Rating Index (mDRI) and modified Klein-Bell [KB] ADL scale [37], or the ADL staircase (Katz ADL Index extended by further IADL items) [28].

\section{Mobility status}

Mobility status was assessed in seven studies (29.2\%; 5 exercise interventions [35, 38, 41, 43, 45] and 2 multidisciplinary programs $[28,30])$. Nine different motor performance measures were identified, including the modified Iowa Level of Assistance Scale (mILOAS) [41, 43], the Timed Up and Go (TUG) [35, 41], the walking item of the Clinical Outcome Variables Scale (COVS) $[28,29]$, the Short Physical Performance Battery (SPPB) [30], a lower extremity handheld dynamometry strength measurement [45], the One Leg Stance (OLS) and 30-s Chair Stand Test (30CST) [45], the 10-Meter Walking Test (10MWT) [35], the 6-Minute Walk Test (6MWT) [38], and a self-developed postoperative patient activity scale (PPAS) [35]. PA measures were reported in only two studies, including the self-administered University of California, Los Angeles Activity (UCLA) scale [35] or an accelerometer-based PA monitor (activPAL) [31].

\section{Hospital outcomes}

Hospital outcomes were assessed in six studies (25.0\%; 5 multidisciplinary programs $[28,47,49,51,53]$ and 1 exercise intervention [44]). LOS was reported in all these studies. Further outcome measures included discharge destination [28, 47, 51] or hospital costs and other process-of-care measures (e.g., physical therapy consults, orders for bed rest) [47]. 


\section{Adverse clinical events}

Three studies (12.5\%; 3 multidisciplinary programs) assessed mortality $[46,53]$, different complications during hospitalization [53], or falls/fall-related outcomes (Abbreviated Injury Scale [AIS]) [27].

\section{Psychological status}

Psychological factors were assessed in three studies (12.5\%; 2 multidisciplinary programs $[26,52]$ and 1 exercise intervention [36]), using the Geriatric Depression Scale (GDS) [26], the 15D HRQOL questionnaire [52], or the Self-Efficacy Gauge and Life-Satisfaction Index [36].

\section{Cognitive functioning}

Two studies (8.3\%; 1 exercise intervention [39] and 1 multidisciplinary programs [26]) used the Confusion Assessment Method (CAM) to assess the number of delirious patients [39] or the Organic Brain Syndrome (OBS) scale to screen for the number of delirious days during hospitalization and the Mini-Mental State Examination (MMSE) to screen the global cognitive status [26].

\section{Matching of outcome measures}

Table 3 presents the results of the matching procedure and the intervention effects reported for each outcome measure identified among studies. In the following, the results of the matching procedure were initially summarized for each outcome category.

\section{Functional status}

Most frequently, functional measures matched the intervention contents only to a limited extent with items not part of the functional intervention component (e.g., Katz ADL Index $\rightarrow$ only basic transfer and ambulation training) $[28,32,34,36,37,40,42]$. Functional measures that specifically addressed the functional intervention contents (e.g., Katz ADL Index $\rightarrow$ ADL training to improve bathing/dressing, mobility/transferring, toileting, feeding) were used in only three studies $[37,48,50]$. In another three studies, we identified functional measures that did not directly match the interventions, which did not include a functional training component (e.g., Lawton IADL scale $\rightarrow$ no IADL training content) [33, 35, 42].

Six studies suggested ceiling effects for at least one of their functional measures, with $>15 \%$ of participants reaching a score within the best $15 \%$ of the rating scales (Katz ADL Index [50], Barthel Index [40], ADL staircase [28]), or mean scores of the sample within the best $15 \%$ of the rating scale (Barthel Index [42], Katz ADL Index [34], Lawton IADL scale [35]). A missing data rate of $\geq 15 \%$ for functional measures were reported in two studies, which did not present any data for the Lawton
IADL scale [35] or incomplete data for the ADL staircase (only ADL items presented) [28] at discharge.

Two studies used the Lawton IADL scale [35, 42], which did not match to the acute care hospital setting with inappropriate items addressing instrumental home or community activities such as washing, housekeeping, or shopping. Two studies used functional measures (mDRI [37], ADL staircase [28]) that matched to the acute care hospital setting only to a limited extent, including both setting-specific basic ADL items but also setting non-specific IADL items.

\section{Mobility status}

Most frequently, mobility measures specifically matched the mobility intervention component (e.g., 6MWT $\rightarrow$ lower limb endurance training) [28, 30, 38, 41, 43]. Limited matches in which the mobility measure covered the mobility intervention component only to a limited extent (e.g., OLS $\rightarrow$ chair-based pedal exercises; mILOAS transfer, step negotiation and ambulation items $\rightarrow$ only walking training) were found in four studies [31, 35, 43, 45].

Only one study suggested a floor effect, with almost one fourth $(23.3 \%)$ of the total sample reaching a score within the worst $15 \%$ of rating scale of the mILOAS step negotiation item [43]. A missing data rate of $\geq 15 \%$ for mobility measures were reported in three studies [31, 35, 43]. Two of them did not present any or incomplete data for the UCLA (missing data: 100\%) [35] or single mILOAS items (missing data: 15\% [transfers]; 21\% [step negotiation] [43]). The other study reported that in $19 \%$ of the sample, sensor-based PA data were missing due to reasons such as sensor removing, technical problems, or medical reasons [31].

Most studies used mobility measures specifically addressing mobility or physical activities that can be appropriately assessed within the acute care hospital setting (e.g., SPPB $\rightarrow$ functional mobility; 10MWT $\rightarrow$ walking) [28, 30, 38, 41, 43, 45].

Only one study used the UCLA to assess PA behavior, which matched to the acute care hospital setting only to a limited extent, with inappropriate response items addressing intensive physical activities (e.g., swimming, bicycling) or impact sports [35] rather than rehabilitationspecific activities.

\section{Hospital outcomes}

Three studies used hospital outcomes (LOS, hospital costs, discharge destination) that specifically addressed their intervention components [47, 49, 51]. All these studies conducted a multidisciplinary program that included multidisciplinary team meetings with individual care planning, comprehensive geriatric assessments, and/or discharge planning. Limited matches were found 
Table 3 Results of the matching procedure and intervention effects reported for each outcome measure

\begin{tabular}{|c|c|c|c|c|c|c|c|}
\hline \multicolumn{2}{|c|}{ Outcome measures } & \multirow[t]{3}{*}{ Study } & \multicolumn{4}{|l|}{ Matching } & \multirow{3}{*}{$\begin{array}{l}\text { Intervention } \\
\text { effects }\end{array}$} \\
\hline \multirow[t]{2}{*}{ Category } & \multirow[t]{2}{*}{ Instrument } & & \multirow[t]{2}{*}{ Intervention } & \multicolumn{2}{|l|}{ Sample } & \multirow[t]{2}{*}{ Setting } & \\
\hline & & & & $\begin{array}{l}\text { Floor/ceiling effects or rare } \\
\text { event }\end{array}$ & $\begin{array}{l}\text { Missing } \\
\text { data }\end{array}$ & & \\
\hline \multirow[t]{13}{*}{$\mathrm{FCT}$} & \multirow{4}{*}{$\begin{array}{l}\text { (modified) Katz ADL } \\
\text { Index }\end{array}$} & Blanc-Bisson 2008 [33] & - & + & + & + & NA \\
\hline & & Brown 2016 [34] & \pm & - & + & + & n.s. \\
\hline & & Counsell 2000 [48] & + & + & + & + & n.s. \\
\hline & & Landefeld 1995 [50] & + & - & + & + & $\uparrow$ \\
\hline & \multirow[t]{3}{*}{ Barthel Index } & Abizanda 2011 [32] & \pm & + & + & + & n.s. \\
\hline & & Jones 2006 [40] & \pm & - & + & + & n.s. \\
\hline & & Nikolaus 1999 [42] & \pm & - & + & + & n.s. \\
\hline & \multirow[t]{2}{*}{ Lawton IADL scale } & Czyzewski 2013 [35] & - & - & - & - & NA \\
\hline & & Nikolaus 1999 [42] & - & + & + & - & n.s. \\
\hline & ADL staircase & $\begin{array}{l}\text { Stenvall 2007, } 2012 \text { [28, } \\
\text { 29] } \\
\text { Lundström } 2007[26,28]\end{array}$ & \pm & - & - & \pm & NA \\
\hline & FIM & Eyres 2005 [36] & \pm & + & + & + & NA \\
\hline & $\mathrm{mDRl}$ & Hagsten 2004 [37] & \pm & NA & + & \pm & n.s. \\
\hline & mKB ADL scale & Hagsten 2004 [37] & + & + & + & \pm & $\uparrow$ \\
\hline \multirow[t]{16}{*}{$\mathrm{MOB}$} & $6 \mathrm{MWT}$ & He 2015 [38] & + & + & + & + & NA \\
\hline & 10MWT & Czyzewski 2013 [35] & \pm & + & + & + & NA \\
\hline & 30CST & Torres-Sanchez 2017 [45] & \pm & + & + & + & $\uparrow$ \\
\hline & \multicolumn{7}{|l|}{ mILOAS } \\
\hline & total score & Kimmel 2016 [41] & + & + & + & + & n.s. \\
\hline & ambulation item & Oldmeadow 2006 [43] & + & NA & + & + & $\uparrow$ \\
\hline & step negotiation item & Oldmeadow 2006 [43] & \pm & - & - & + & n.s. \\
\hline & transfer items & Oldmeadow 2006 [43] & \pm & NA & - & + & $\uparrow$ \\
\hline & activPAL & Taraldsen 2014 [31] & \pm & + & - & + & $\uparrow$ \\
\hline & Handheld dynamometry & Torres-Sanchez 2017 [45] & \pm & + & + & + & $\uparrow$ \\
\hline & OLS & Torres-Sanchez 2017 [45] & \pm & + & + & + & $\uparrow$ \\
\hline & PPAS & Czyzewski 2013 [35] & \pm & NA & + & + & $\uparrow$ \\
\hline & SPPB & Prestmo 2015 [30] & + & + & + & + & $\uparrow$ \\
\hline & TUG & Czyzewski 2013 [35] & \pm & + & + & + & NA \\
\hline & UCLA scale & Czyzewski 2013 [35] & \pm & NA & - & \pm & NA \\
\hline & covs & $\begin{array}{l}\text { Stenvall 2007, } 2012[28, \\
\text { 29] } \\
\text { Lundström } 2007 \text { [26] }\end{array}$ & + & NA & + & + & n.s. \\
\hline \multirow[t]{7}{*}{$\mathrm{HU}$} & \multirow[t]{4}{*}{ LOS } & Barnes 2012 [47] & + & + & + & + & $\uparrow$ \\
\hline & & Huusko 2000 [49] & + & + & + & + & $\uparrow$ \\
\hline & & Siebens 2000 [44] & - & + & + & + & n.s. \\
\hline & & Vidan 2005 [53] & \pm & + & + & + & n.s. \\
\hline & \multirow[t]{2}{*}{ Discharge destination } & Naglie 2002 [51] & + & + & + & + & $\uparrow$ \\
\hline & & Stenvall 2007 [28] & \pm & + & + & + & n.s. \\
\hline & Hospital costs & Barnes 2012 [47] & + & + & + & + & $\uparrow$ \\
\hline \multirow[t]{3}{*}{ ACE } & \multirow[t]{2}{*}{ Medical complications } & Stenvall 2012 [29] & + & NA & + & + & NA \\
\hline & & Vidan 2005 [53] & + & + & + & + & $\uparrow$ \\
\hline & Mortality & Asplund 2000 [46] & \pm & - & + & + & n.s. \\
\hline
\end{tabular}


Table 3 Results of the matching procedure and intervention effects reported for each outcome measure (Continued)

\begin{tabular}{|c|c|c|c|c|c|c|c|}
\hline \multicolumn{2}{|c|}{ Outcome measures } & \multirow[t]{3}{*}{ Study } & \multicolumn{4}{|l|}{ Matching } & \multirow{3}{*}{$\begin{array}{l}\text { Intervention } \\
\text { effects }\end{array}$} \\
\hline \multirow[t]{2}{*}{ Category } & \multirow[t]{2}{*}{ Instrument } & & \multirow[t]{2}{*}{ Intervention } & \multicolumn{2}{|l|}{ Sample } & \multirow[t]{2}{*}{ Setting } & \\
\hline & & & & $\begin{array}{l}\text { Floor/ceiling effects or rare } \\
\text { event }\end{array}$ & $\begin{array}{l}\text { Missing } \\
\text { data }\end{array}$ & & \\
\hline & & Vidan 2005 [53] & \pm & - & + & + & $\uparrow$ \\
\hline & AIS & $\begin{array}{l}\text { Stenvall 2007,2012 [27- } \\
\text { 29] }\end{array}$ & + & - & - & + & $\uparrow$ \\
\hline & Falls & Stenvall 2007 [27] & + & + & + & + & $\uparrow$ \\
\hline \multirow[t]{4}{*}{ PSY } & Self-Efficacy Gauge & Eyres 2005 [36] & \pm & + & + & + & NA \\
\hline & Life Satisfaction Index & Eyres 2005 [36] & - & + & + & + & NA \\
\hline & GDS & Lundström [26] & - & + & - & + & n.s \\
\hline & 15D HRQOL & Pitkälä 2008 [52] & \pm & + & + & + & $\uparrow$ \\
\hline \multirow[t]{3}{*}{ COG } & CAM & Jeffs 2013 & \pm & - & + & + & n.s \\
\hline & OBS scale & Lundström 2007 [26] & + & - & + & + & $\uparrow$ \\
\hline & MMSE & Lundström 2007 [26] & \pm & + & + & + & n.s. \\
\hline
\end{tabular}

6MWT 6-Minute Walk Test, 10MWT 10-Meter Walking Test, 30CST 30-Seconds Chair Stand Test, AIS Abbreviated Injury Scale, CAM Confusion Assessment Method, COVS Clinical Outcome Variables Scale, FIM Functional Independent Measure, GDS Geriatric Depression Scale, HRQOL Health-related Quality of Life, LOS Length of stay, $m D R I$ modified Disability Rating Index, mILOAS modified lowa Level of Assistance Scale, mKB ADL scale modified Klein-Bell ADL scale, MMSE Mini-Mental State Examination, OBS scale Organic Brain Syndrome scale, OLS One Leg Stance, PPAS Postoperative Patient Activity Scale, SPPB Short Physical Performance Battery, TUG Timed Up and Go, UCLA scale University of California, Los Angeles Activity scale

+ , "match"; \pm , "limited match"; -, "no match"; NA, not available; $\uparrow$, significant between-group differences in favor of the intervention group ( $p \leq 0.05$ ); n.s., no significant between-group differences in favor of the intervention group $(p>0.05)$

for two other multidisciplinary intervention studies which assessed LOS [53] or discharge destination [28]; however, without including specific discharge planning procedures within their multidisciplinary program. No match was found for one study, which was the only one that assessed the unspecific effect of an additional exercise intervention on a hospital outcome (LOS) [44].

Ceiling and floor effects or rare events were not apparent for any of these setting-specific hospital outcomes, and none of the six studies reported missing data.

\section{Adverse clinical events}

Two studies analyzing adverse clinical events used outcome measures that specifically matched to the intervention. Both of them assessed the incidence of medical complications during hospitalization to evaluate the specific effect of their intervention contents focusing on the identification, prevention and treatment of these complications $[29,53]$. One of these studies also assessed the effect of a systematic assessment and treatment of fall risk factors by the number of falls/fallers and the AIS that specifically matched to this specific intervention component $[27,29]$. Two studies assessed mortality during hospitalization, which were addressed to a limited extent by the increased, multidisciplinary diagnostic progress, the improved therapeutic care planning, and the increased patient contact time during acute hospitalization $[46,53]$.

In both studies assessing mortality, a mortality rate of only $3 \%$ during hospitalization was observed $[46,53]$, indicating a rare event. The AIS used to assess fall-related injury severity showed a ceiling effect with $42 \%$ of fallers reaching the best possible AIS score and missing data for $81 \%$ of participants who had not fallen [27]. For medical complications, falls, and mortality, no missing data were reported in all studies $[27,46,53]$.

Adverse clinical events were appropriately assessed based on nursing/medical records or patient charts in all studies [27, 29, 46, 53].

\section{Psychological status}

None of the studies focusing on psychological status used a psychological measure that specifically matched their intervention contents [26, 36, 52]. Limited matches were found in two studies, using the 15D HRQOL with single items that were addressed by the intervention contents (15D HRQOL mobility dimension $\rightarrow$ physiotherapy, 15D HRQOL mental function dimension $\rightarrow$ orientation training) [52] or the Self-Efficacy Gauge, which has been specifically developed to assess selfperceived confidence in occupational performances, to evaluate an additional occupational therapy program [36]. Psychological measures (Life-Satisfaction Index [36], GDS [26]) that did not match a specific content of their interventions were found in two studies.

Ceiling or floor effects were not identified for any psychological measure [26, 36, 52], and only one study reported a missing data rate of $20 \%$ for the GDS at discharge [26].

All psychological measures used in the studies addressed constructs that can be appropriately assessed within the acute care hospital setting. 


\section{Cognitive functioning}

In one of the two studies analyzing cognitive functioning, the number of delirious days as assessed by the OBS scale specifically matched the intervention contents of active preventing, detecting, and treating delirium [26]. The same study also used the MMSE, which matched this intervention component only to a limited extent not including any further cognitive training contents [26]. In the other study, the CAM also only to a limited extent matched in evaluating the effect of additional orientation exercises on the number of delirious patients [39].

For the number of delirious days, a ceiling effect was identified, with $65 \%$ of patients having no delirious day [26], and the number of delirious patients represented a rare event, with only $5.4 \%$ of patients having a delirium episode during hospitalization [39].

All cognitive measures could be rated as appropriate for use in the acute care hospital setting.

\section{Intervention effects in relation to the matches}

In the following, the main findings reported on the primary outcomes were related to the results of the matching procedure. Details on the intervention effects on the outcome measures identified among studies can be found in Table 3.

\section{Functional status}

Seven studies (4 exercise interventions [32, 34, 37, 40, $42]$ and 2 multidisciplinary programs $[48,50]$ ) reported on between-group differences in functional status at hospital discharge, whereas four studies (3 exercise interventions $[33,35,36]$ and one multidisciplinary programs [28]) did not. In those studies $(n=5)$ with no or only limited matches between functional measures and exercise intervention, no significant benefits of the intervention could be documented [32, 34, 37, 40, 42]. Only in those two studies where the functional measures specifically addressed the exercise intervention [37], or an intervention component of the multidisciplinary program [50], a significant superior effect of the intervention on the functional status was identified.

\section{Mobility status}

Six studies (5 multidisciplinary programs $[28,47,49,51$, 53] and 1 exercise intervention [44]) reported on between-group differences in mobility status after surgery or at hospital discharge based on a variety of 11 different mobility measures. One study only analyzed within-group changes for the mobility outcomes at hospital discharge [38].

Out of the four mobility measures with interventionspecific matches, two (SPPB, mILOAS ambulation item) revealed a significant benefit of the additional exercise intervention [43] or the multidisciplinary program [30] over the usual care on motor performance, whereas the other two did not (COVS walking item [28], mILOAS [41]). All other seven mobility measures with limited intervention-related matches (handheld dynamometry, OLS, 30CST, mILOAS step negotiation and transfer items, PPAS, activPAL) revealed significant beneficial effects in the experimental groups (3 exercise interventions [35, 43, 45] and 1 multidisciplinary program [31]), except for one (mILOAS step negotiation) [43].

Out of the mobility measures that did not reveal significant between-group differences, two covered single subjective rating items of more comprehensive assessment scales (COVS walking item, mILOAS step negotiation item) [28, 43], with partly floor effects in the sample (mILOAS step negotiation item) [43], and one was a comprehensive assessment scale combining subjective rating and objectively-measured items (mILOAS total score) [41].

\section{Hospital outcomes}

Six studies (5 multidisciplinary programs $[28,47,49,51$, 53] and 1 exercise intervention [44]) analyzed betweengroup differences in LOS, discharge destination, and/or hospital costs at hospital discharge. Significantly shorter LOS, more patients reintegrated into the community, and lower hospital costs among the intervention group were found only for these three studies in which the hospital outcomes specifically matched the intervention components of the multidisciplinary programs $[47,49$, 51]. No significant between-group differences could be documented [28] in multidisciplinary studies with only limited matches between the hospital outcomes (LOS, discharge destination) and their intervention components [28] and in the exercise intervention study showing no match [44].

\section{Adverse clinical events}

Between-group differences in adverse clinical events at hospital discharge were analyzed in three multidisciplinary intervention studies [29, 46, 53]. Two studies assessing adverse clinical events that specifically matched their intervention components reported a significant lower number of falls, fallers and minor to moderate fall-related injuries [27] and reduced medical complications in favor of the intervention [53]. Out of the two studies that analyzed (also) mortality, which matched as an outcome measures only to a limited extent to the multidisciplinary interventions during early inpatient rehabilitation in the acute care hospital setting, one reported a significant effect of their intervention in reducing mortality during hospitalization [53], whereas the other study did not [46]. 


\section{Psychological status}

Two multidisciplinary studies analyzed between-group differences in HRQOL [52] and/or depression [26] at hospital discharge. In these two studies, a significant psychological benefit of the intervention compared to usual care was observed only by using the 15D HRQOL that showed a limited match, with single dimensions specifically addressing an intervention component [26, 52]. The GDS, as used in one of these studies, did not match the intervention and revealed no significant between-group differences [26].

\section{Cognitive functioning}

Two studies (1 multidisciplinary program [26] and 1 exercise intervention [39]) analyzed between-group differences in cognitive functioning during hospitalization. For the cognitive measures with limited matches to the intervention (CAM [delirious patients], MMSE), both studies reported no significant benefit of the intervention compared to the usual care [26,39]. Only for the number of delirious days as assessed by the OBS scale, which specifically matched the intervention component of active prevention, detection and treatment of delirium within the multidisciplinary program, significant between-group differences in favor of the intervention group were reported [26].

\section{Discussion}

The aim of this review was to analyze the matching of outcome measures used in previous RCTs on early rehabilitation in acutely hospitalized older patients to the specific study characteristics (intervention, sample, and setting) and to evaluate the effects of matching on the main findings reported in these RCTs. In the 24 studies included in this review, the selection of primary outcome measures differed considerably, with a total of 33 different outcome measures across six different outcome categories. The matching process indicated also a large heterogeneity in the appropriateness of the selected outcome measures for the intervention contents, the study sample, and the acute geriatric hospital setting. Our findings suggest that a good match especially between the outcome measure and the intervention contents seems to have increased the likelihood for documenting significant intervention-induced benefits among the included studies.

\section{Functional status}

Functional status defined as (I) ADL functioning has become a key outcome during hospitalization in older patients [55]. The ability to perform (I) ADL is a crucial part of human functioning, disability and health, as located centrally in the model of the International
Classification of Functioning, Disability and Health (ICF) from the World Health Organization [56], and a major established outcome for rehabilitation. It was therefore not surprising that the primary outcome measures most frequently used in the included studies focused on (I)ADL. However, there was a large heterogeneity in assessing (I) ADL functioning, with seven different (I) ADL instruments identified among the studies. Our findings supports the lack of consensus regarding measuring the functional status of acutely hospitalized older patients in clinical research, as previously reported in a systematic review on the variability of (I) ADL measures in this patient population [57].

Most frequently, the various functional measures addressed ADL rather than IADL. This might be related to the fact that ADL measures assess basic activities essential for an individual's direct self-care (e.g., bathing, dressing, walking) which are primarily targeted by treatments during the early rehabilitation phase in the acute care hospital setting. In contrast, IADL measures assess more complex activities that are not necessarily a precondition for basic functions, but that are more concerned with self-reliant functioning in the home (e.g., food preparation, housekeeping) or community environment (e.g., shopping, transportation), being rather addressed in the later rehabilitation phases or after hospital discharge. None of the studies using an IADL measure specifically targeted such home or community activities by their intervention [35, 42]. Based on these mismatches of IADL measures with the acute care hospital setting and the intervention contents, none of these studies reported favorable IADL outcomes for their intervention groups $[35,42]$. The majority of the studies with a primary IADL or a combined (I) ADL measure even did not present any data for the IADL measures [35] or analyzed only ADL items but not IADL items of the combined (I) ADL measure at hospital [28], which might suggest that IADL functioning was not assessed, potentially also due to the mismatch of measuring IADL in the acute care hospital setting, as discussed before.

For studies using ADL measures, we predominantly found only limited matches between these instruments and the intervention contents [28, 32, 34, 36, 37, 40, 42]. None of these studies revealed a beneficial intervention effect on the functional status. This might be related to the fact that their interventions specifically addressed only a limited number of ADL items such as transferring, walking, or bathing; while other items (e.g., bowel and bladder control), which show limited responsiveness to available interventions, were not addressed. Even if a beneficial effect on addressed items occurred, the impact on ADL instrument's overall scores, as analyzed in all these studies, might have been too small to reveal significant benefits related to the intervention. 
The only two studies reporting better ADL functioning in their intervention groups at discharge used modified ADL instruments, excluding the items that were not contents of their interventions (e.g. eating, incontinence) $[37,50]$. Such modifications may increase the specificity and sensitivity of the outcome measure and, in turn, seem to increase the probability to capture significant intervention effects, as suggested by the significant findings of the two studies. However, it must be kept in mind that modified assessment instruments are no longer validated, thus requiring further psychometric testing before their application [16].

Another potential explanation for insufficient intervention effects on (I) ADL functioning might be related to the ceiling effects identified for most of the ADL instruments already at hospital admission (Barthel Index [40], (modified) Katz ADL Index [34, 50], ADL staircase [28], Lawton IADL scale [35]), indicating a mismatch between these instruments and the characteristics of the sample. If patients' scores are close to the top of the scale (i.e. at the ceiling) already at baseline, there is only little room for further subsequent improvements, substantially reducing an instrument's sensitivity as well as a study's ability to detect significant changes in those patients $[14,58]$. As already recommended previously [8], future studies may therefore use functional measures that cover a broader range of ability levels for acutely hospitalized older patients to explore the effects of early rehabilitation in this highly heterogeneous patient population.

\section{Mobility status}

Mobility is fundamental to healthy aging and quality of life in older adults [59], and a loss of mobility can result in a decline in autonomy [60]. Consequently, measuring mobility can determine the level of independence and the health care needs in the older population [61]. Measures addressing the patients' mobility status formed the second largest category of primary outcome measures. Surprisingly, we identified an even greater heterogeneity of instruments on mobility status than reported above for functional status. None of the primary mobility instruments was used in more than one study, except for the mILOAS. However, also the mILOAS was used differently in two studies, analyzing either the total score [41] or only individual items (walking, step negotiation, transfers) [43]. Our findings on this heterogeneity are in line with a previous systematic review on instruments used to evaluate mobility of older patients during hospitalization [62], highlighting that the lack of consensus not only includes functional but also mobility measure in this setting.

For none of the mobility measures, we identified a total mismatch with a study's intervention contents, probably based on the fact that this review considered only studies which included a physical exercise intervention [32-45] or a multidisciplinary program with physical exercise as a training component [27, 30, 46-53]. Even if the specific physical intervention content was not directly matched by most of the mobility measures - for example, in terms of conducting physical exercise on specific motor abilities (e.g., pedal exercise $\rightarrow$ endurance) but assessing other motor abilities (e.g., OLS $\rightarrow$ balance) - both the mobility measure and the intervention content were related to the overarching construct of mobility, leading to at least limited matches between those. Most frequently, these mobility measures with limited intervention-specific matches still revealed significant effects in favor of the intervention groups compared to the usual care groups. This finding suggests that mobility measures seem to be more sensitive to detect potential intervention-induced effects than the functional measures discussed above, for which a rather high specificity ("perfect match") to the intervention content was required to reveal such significant between-group differences.

Another advantage of the mobility measures and rationale for their higher potential to detect interventioninduced changes compared to the functional measures might be seen in their coverage of a broader spectrum of patients' abilities in the highly heterogeneous population of older patients. We identified no ceiling or floor effects for primary mobility measures, except for one study reporting a floor effect for a single item of the mILOAS (negotiation item) [43]. However, no floor effects occurred when its total score was used, as reported in another study [41].

Considering the instrument format of the mobility measures used in the studies analyzing between-group differences (i.e. subjective, observation-based or more standardized, objective measurement methods), it is conspicuous that those measures which did not reveal intervention effects were based on subjective, observationbased rating items (COVS walking item [28], mILOAS step negotiation item [43]) or a more comprehensive assessment scale including predominantly subjective items (mILOAS) [41]. In contrast, all objective mobility measures, for which between-group differences were analyzed (SPPB, handheld dynamometry, OLS, 30CST, mILOAS ambulation item [walking distance], activPAL), revealed favorable mobility outcomes for the intervention group [30, 43, 45], suggesting that this instrument format seems to be more sensitive to show the benefit of exercise-based interventions.

The mobility measures most frequently used addressed key motor functions such as standing, walking, and/or transferring (e.g., SPPB, 10MWT, 30CST, TUG) [30, 35, 45], which are crucial for functional mobility and 
independence in daily life [62, 63]. PA behavior as a more complex, multidimensional construct was primarily investigated in only 2 studies (UCLA [35], activPAL [31]), with only one of them presenting PA data at discharge [31]. This study revealed a positive intervention effect on PA behavior assessed by a sensor-based PA monitor. Using such highly objective PA assessment instruments might be a promising approach to demonstrate intervention-induced effects; however, it might also be associated with feasibility issues in the sample of older patients, as a high missing data rate was reported in this study (19\%). As indicated in a previous review on the utility and accuracy of PA sensors in older hospitalized patients, further research is required to examine their feasibility as well as their validity in this patient population [64].

\section{Hospital outcomes}

LOS, hospital costs, or discharge destination are outcomes associated with healthcare utilization or medical service use in a broad sense and are related to a series of potential cost-saving factors for healthcare [65]. For example, a reduction of LOS can decrease inpatient hospital costs and increase hospital bed availability, increasing the overall cost-efficiency of hospitals [66]. Given the great importance of such cost-related outcomes, it was not surprising that they were the third largest category of primary outcomes identified in this review. LOS was the most frequently evaluated hospital outcome, which might be related to the fact that this hospital outcome may be considered as the key driver of inpatient costs [38] and as an indicator of hospital efficiency [67].

Within our matching procedure, it was initially assumed that changes in hospital outcomes require an optimized organizational proceeding between different inhospital disciplines, i.e. a multidisciplinary intervention program. This assumption was based on previous findings made by de Morton (2007), suggesting that improvements in these outcomes might result from a better coordination of care provision, increased medical, nursing or allied health interventions, a combination of improved team goal setting and discharge planning, and/ or increased patient contact time during acute hospitalization [8]. Therefore, matches or limited matches between hospital outcomes and intervention contents were given only for multidisciplinary studies. Among these multidisciplinary studies, however, only those with intervention contents strictly optimized to the hospital outcome (e.g., discharge destination $\rightarrow$ discharge planning) revealed significant interventioninduced benefits [47, 49,51]. All other multidisciplinary studies that used hospital outcomes with only limited matches to the intervention contents (e.g., discharge destination $\rightarrow$ only individual care planning but no specific discharge planning) could not document such beneficial effects [28, 53]. The only study evaluating an exercise-only intervention by using LOS as a primary outcome [44], which resulted in a mismatch with the intervention contents, was unable to detect significant between-group differences. Hospital outcomes seem not to be sufficiently specific and sensitive enough to document unspecific effects of an exercise intervention and may therefore not be considered as the first choice for the evaluation of interventions with a mere exercise focus in the acute geriatric hospital setting [9]. Our findings support the initial assumption that hospital outcomes might be able to reveal benefits of multidisciplinary programs; however, only if the intervention contents were specifically addressed by the intervention contents.

On the other hand, hospital outcomes are based on a simple data acquisition with high specificity to the hospital setting, as indicated by the overall lack of missing data in all the studies primarily analyzing hospital outcomes [28, 44, 47, 49, 51, 53]. Outcomes such as LOS, hospital costs, or discharge destination are usually based on highly objective, reliable and precise data, which are already captured within the routine hospital records, requiring only little additional effort for data acquisition.

\section{Adverse clinical events}

An adverse clinical event can generally be described as an acute clinical problem that newly occurred during hospitalization and was not present at hospital admission [68]. According to previous systematic reviews on the effects of physical exercise intervention in acutely hospitalized older patients $[8,63]$, the identified outcome measures such as falls, medical complications, and mortality were categorized as clinical adverse events also in this review. This category of outcome measures stands out as it does not focus on functioning and disability following the established rehabilitation paradigm of the ICF framework [56] but rather focuses on patients' acute clinical problems and medical conditions. This might also provide a reasonable explanation for the non-frequent use of primary outcome measures out of this category. If adverse clinical events were investigated in the included studies, they were most frequently (6 out of 9 studies) defined as a secondary outcome [34, 40, 41, $44,47,48]$, and only three studies, defined them as a primary outcome $[27,46,53]$, with all of them evaluating multidisciplinary program.

More or less, all outcome measures of this category represent rather rare events (e.g., injuries falls, mortality), with the consequence that even in high-risk groups for such outcomes, it may need very large sample sizes and/or highly specific and extraordinary effective intervention 
strategies to reveal significant improvements over the limited time period of acute care hospitalization. In addition, adverse clinical events can be related to a variety of different factors such as system failures, involuntary errors, or negligence [69]. A multidisciplinary approach was therefore considered to be an essential basic requirement for a match between the outcome category of adverse clinical events and the intervention. In studies analyzing the effects of a multidisciplinary program on medical complications or falls, the intervention contents were indeed strictly optimized to reduce such adverse clinical events (e.g., treatment of fall risk factors $\rightarrow$ number of falls; identification, prevention and treatment of complications $\rightarrow$ postoperative complications), leading to significant benefits induced by their multidisciplinary programs compared to usual care $[29,53]$.

Mortality was used as a primary outcome in two multidisciplinary studies [46, 53]. Reducing mortality is certainly one of the most desirable goals in clinical health care. Mortality can be easily, objectively and reliably measured, as also indicated by lack of missing data among these two studies $[46,53]$. However, it can also be described as the "hardest outcome of all", as mortality rates can be affected by many factors other than the contents or quality of clinical care [70] that cannot all be controlled for in a RCT. Based on the complexity of mortality, only limited matches to the intervention approach with primary focus on functional rehabilitation had been achieved in both studies, even if the multidisciplinary programs included intervention contents that might be beneficial for preventing mortality (e.g., increased patient contact time, multidisciplinary diagnostic progress). The very low mortality rates $(<3 \%)$ emphasize the assumption that mortality fortunately represents a rare event, even in the high-risk group of acutely hospitalized older patients. To allow for the documentation of a successful intervention on such rare events, large sample sizes combined with highly effective intervention strategies are required to allow for documentation of a successful intervention. Based on low mortality rates and the limited matches to the interventions, it was surprising that one of them reported a significant between-group difference in favor of their intervention group [53]. However, as also mentioned by the authors of this study, this finding has to be interpreted with caution. Although the relative interventioninduced reduction in mortality seems huge (-89\%), because the absolute number of deaths was low in both groups (control group: $n=9$ vs. intervention group: $n=1$ ), they could not formally exclude that this between-group difference was due to chance.

\section{Psychological status}

The psychological measures used as primary outcomes addressed different psychological constructs such as depression, self-efficacy, life satisfaction, or quality of life. Only three studies defined such measures as a primary outcome, indicating that psychological constructs were not a main focus of the studies identified in this review. None of the interventions of the studies with a primary psychological measure had a clear interventional approach to target psychological factors [26, 36, 52], suggesting that in these studies it was assumed that intervention contents might be indirectly associated with relevant psychological side effects. Out of the 2 studies analyzing between-group differences in psychological outcomes $[26,36,52]$, only one study revealed a psychological benefit of the intervention. The fact that this study used a multidimensional psychological measure (15D HRQOL) with dimensions (e.g., mobility, mental function) that addressed some intervention contents at least to a limited extent (e.g., psychotherapy, orientation training) might explain this rather unspecific effect [52]. The other study could not document interventioninduced psychological benefits, which might be a direct consequence of the mismatch between the selected psychological outcome measure (GDS) and the intervention program [26].

\section{Cognitive functioning}

Cognitive functioning also was not a main focus of the identified studies, as only two of them defined global cognitive status (MMSE) and/or delirium (OBS scale, CAM) as a primary outcome [26, 39]. Among these two studies, only the specific multidisciplinary intervention with focus on active prevention, detection and treatment of delirium showed beneficial effects [26]. The same study was, however, not able to document interventioninduced effects on the patients' global cognitive status, which may be related to the fact that in addition the delirium-related, acute cognitive intervention contents, the multidisciplinary program included no further cognitive intervention contents that specifically addressed cognitive functioning more globally as assessed by the MMSE.

The other study could not document an interventioninduced effect on the number of delirious patients as assessed by the CAM during hospitalization; however, the intervention of this study only included a cognitive intervention content that seemed not specific enough for delirium treatment, in terms of an orientation program [39]. Another potential explanation might be the low incident of delirium in the sample of this study $(<6 \%)$, reducing the power to detect a significant intervention effect, especially when having in mind that in such rare events highly specific and effective intervention strategies are required to reach significance. The study reporting beneficial effects on delirium showed also a ceiling effect, with more than half of participants 
(65\%) having no delirious day during hospitalization [26]; however, the more specific delirium-related intervention contents and the selection of a nondichotomous, more sensitive scaling procedure for delirium (number of delirious days vs. delirious patients) might have still led to significant intervention effects. The lack of significant intervention effects documented by the MMSE [26] and the CAM [39] might also be related to their instrument type. Both were primarily developed as screening instruments, either for global cognitive functioning (MMSE) or for delirium (CAM), which may have limited the sensitivity of these instruments to detect intervention-induced changes among these two studies.

\section{Limitations}

This review has some limitations. First, the matching procedure was based on subjective appraisals of the authors; however, standardized criteria were used which were derived from recommended guidelines [14]. To our knowledge, this review is the first to evaluate the selection of outcome measures in studies on early rehabilitation in the acute care hospital setting by such criteria, representing the most innovative feature of this review. Second, due to the international nature of this review and the inherent differences in the health care systems of the countries in which the studies were conducted, it was sometimes difficult to determine if the study took place in the acute care hospital setting. Consequently, the selection process might be affected by inconsistent terminology of the acute care hospital setting among different countries. Third, the main findings of this review were related to the primary outcome measures identified among the included studies. A clear definition of the study's primary outcome measures in the method section of the included articles was sometimes lacking. The identification of the primary outcome measures was therefore based on the researchers' critical appraisal of the information provided in the articles, considering especially the study aims mentioned in the articles. The identification of the primary outcome measures was also performed independently by two researches with disagreements resolved by consensus or third party consultation. Fourth, only information provided in the included articles was evaluated in this review, although the authors may have used additional or more detailed methodology not stated or unclearly described in the articles.

\section{Conclusions}

The present systematic review provided for the first time a detailed overview and critical appraisal of the primary outcome measures used in previous RCTs to evaluate early inpatient rehabilitation for acutely hospitalized older patients. Current findings highlight that the matching of the outcome measures with especially the contents of the intervention to be evaluated represents a key factor to reveal significant benefits attributable to the intervention. Among the different categories of outcome measures, those assessing the mobility status seem to be more sensitive to intervention-induced effects of early rehabilitation programs than those assessing the functional, psychological or cognitive status, hospital outcomes, or adverse clinical events. For future studies, it is recommended to identify not only outcome measures with established psychometric properties in the different sub-samples of the acute geriatric hospital setting, but also to select outcome measures that match the specific intervention contents. Inconsistent findings on the effectiveness of early rehabilitation programs in this setting might have been partly due to the inappropriate selection of outcome measures.

\section{Additional files}

Additional file 1: Table S1. Search strategy used in PubMed.
(DOCX $15 \mathrm{~kb})$

Additional file 2: PRISMA checklist. (DOCX $31 \mathrm{~kb}$ )

Additional file 3: Table S2. Methodological quality scores on the PEDro scale for each included study. (DOCX $56 \mathrm{~kb}$ )

\begin{abstract}
Abbreviations
(I)ADL: (instrumental) Activities of Daily Living; 10MWT: 10-Meter Walking Test; 30CST: 30-seconds Chair Stand Test; 6MWT: 6-Minute Walk Test; AIS: Abbreviated Injury Scale; CAM: Confusion Assessment Method; COPD: Chronic Obstructive Pulmonary Disease; COVS: Clinical Outcome Variables Scale; FIM: Functional Independence Measure; GDS: Geriatric Depression Scale; HRQOL: Health-Related Quality of Life; ICF: International Classification of Functioning, Disability and Health; LOS: Length of stay; mDRI: Modified Disability Rating Index; mILOAS: Modified lowa Level of Assistance Scale; mKB ADL scale: Modified Klein-Bell ADL scale; MMSE: MiniMental State Examination; OBS: Organic Brain Syndrome Scale; OLS: One Leg Stance; PA: Physical activity; PPAS: Self-developed postoperative patient activity scale; RCTs: Randomized controlled trials; SPPB: Short Physical Performance Battery; TUG: Timed Up and Go; UCLA scale: University of California, Los Angeles Activity scale
\end{abstract}

\section{Acknowledgements}

We acknowledge financial support by Deutsche Forschungsgemeinschaft within the funding programme Open Access Publishing, by the BadenWürttemberg Ministry of Science, Research and the Arts and by RuprechtKarls-Universität Heidelberg.

\section{Authors' contributions}

$\mathrm{KH}, \mathrm{JMB}, \mathrm{PH}$, and $\mathrm{CW}$ conceived and designed the review. $\mathrm{PH}, \mathrm{NB}$, and $\mathrm{CW}$ completed acquisition of data. All authors analyzed and interpreted the data and were involved in drafting and critical revision of the manuscript. All authors have read and approved the final manuscript.

\section{Funding}

The study was supported by the Robert Bosch Foundation and the Network Aging Research (NAR) at the Heidelberg University. The funding sources had no role in the design and conduct of the study; collection, management, analysis, and interpretation of the data; and preparation, review, or approval of the manuscript. 


\section{Availability of data and materials}

All data were retrieved from published RCTs and extracted in Table 2. The exact references can be found in the list of references. The relevant data supporting the conclusions of this review are included within this article and its additional files.

\section{Ethics approval and consent to participate Not applicable.}

\section{Consent for publication}

Not applicable.

\section{Competing interests}

The authors declare that they have no competing interests.

\section{Author details}

${ }^{1}$ Network Aging Research (NAR), Heidelberg University, Bergheimer Str. 20, 69115 Heidelberg, Germany. ${ }^{2}$ Agaplesion Bethanien Hospital Heidelberg, Geriatric Center at the Heidelberg University, Heidelberg, Germany. ${ }^{3}$ Center for Geriatric Medicine, Heidelberg University, Heidelberg, Germany.

Received: 10 January 2019 Accepted: 2 July 2019

\section{Published online: 09 July 2019}

\section{References}

1. Buurman BM, Hoogerduijn JG, de Haan RJ, Abu-Hanna A, Lagaay AM, Verhaar HJ, Schuurmans MJ, Levi M, de Rooij SE. Geriatric conditions in acutely hospitalized older patients: prevalence and one-year survival and functional decline. PLoS One. 2011;6(11):e26951.

2. Zisberg A, Shadmi E, Sinoff G, Gur-Yaish N, Srulovici E, Admi H. Low mobility during hospitalization and functional decline in older adults. J Am Geriatr Soc. 2011;59(2):266-73.

3. Martínez-Velilla N, Cadore E, Casas-Herrero Á, Idoate-Saralegui F, Izquierdo M. Physical activity and early rehabilitation in hospitalized elderly medical patients: systematic review of randomized clinical trials. J Nutr Health Aging. 2016;20(7):738-51.

4. Fortinsky RH, Covinsky KE, Palmer RM, Landefeld CS. Effects of functional status changes before and during hospitalization on nursing home admission of older adults. J Gerontol A Biol Sci Med Sci. 1999;54(10):M521-6.

5. Brown CJ, Friedkin RJ, Inouye SK. Prevalence and outcomes of low mobility in hospitalized older patients. J Am Geriatr Soc. 2004;52(8):1263-70.

6. Covinsky KE, King JT Jr, Quinn LM, Siddique R, Palmer R, Kresevic DM, Fortinsky RH, Kowal J, Landefeld CS. Do acute care for elders units increase hospital costs? A cost analysis using the hospital perspective. J Am Geriatr Soc. 1997:45(6):729-34.

7. Kortebein P. Rehabilitation for hospital-associated deconditioning. Am 」 Phys Med Rehabil. 2009;88(1):66-77.

8. De Morton NA, Keating JL, Jeffs K. Exercise for acutely hospitalised older medical patients. Cochrane Database Syst Rev. 2007;1:CD005955.

9. Kanach FA, Pastva AM, Hall KS, Pavon JM, Morey MC, Morey M. Effects of structured exercise interventions for older adults hospitalized with acute medical illness: a systematic review. J Aging Phys Act. 2018;26(2):284-303.

10. Kosse NM, Dutmer AL, Dasenbrock L, Bauer JM, Lamoth CJC. Effectiveness and feasibility of early physical rehabilitation programs for geriatric hospitalized patients: a systematic review. BMC Geriatr. 2013;13(1):107.

11. McKelvie S, Hall AM, Richmond HR, Finnegan S, Lasserson D. Improving the rehabilitation of older people after emergency hospital admission. Maturitas. 2018;111:20-30.

12. de Morton NA, Keating JL, Jeffs $K$. The effect of exercise on outcomes for older acute medical inpatients compared with control or alternative treatments: a systematic review of randomized controlled trials. Clin Rehabil. 2007;21(1):3-16.

13. Scheerman $K$, Raaijmakers $K$, Otten RHJ, Meskers CGM, Maier AB. Effect of physical interventions on physical performance and physical activity in older patients during hospitalization: a systematic review. BMC Geriatr. 2018;18(1):288.

14. Coster WJ. Making the best match: selecting outcome measures for clinical trials and outcome studies. Am J Occup Ther. 2013;67(2):162-70.

15. Clarke M, Williamson PR. Core outcome sets and systematic reviews. Syst Rev. 2016;5(1):5-8.
16. Wales K, Clemson L, Lannin N, Cameron I. Functional assessments used by occupational therapists with older adults at risk of activity and participation limitations: a systematic review. PLoS One. 2016;11(2):1-20.

17. Tyson S, Connell L. The psychometric properties and clinical utility of measures of walking and mobility in neurological conditions: a systematic review. Clin Rehabil. 2009;23(11):1018-33.

18. Greenhalgh J, Long AF, Brettle AJ, Grant MJ. Reviewing and selecting outcome measures for use in routine practice. J Eval Clin Pract. 1998;4(4): $339-50$.

19. Higgins JGS. Cochrane handbook for systematic reviews of interventions. Chichester: John Wiley \& Sons; 2011.

20. Moher D, Liberati A, Tetzlaff J, Altman DG. The PG: preferred reporting items for systematic reviews and meta-analyses: the PRISMA statement. PLoS Med. 2009;6(7):e1000097.

21. EPOC: Effective Practice and Organisation of Care (EPOC). Data collection form. EPOC Resources for review authors. Oslo: Norwegian Knowledge Centre for the Health Services; 2013.

22. McHorney CA, Tarlov AR. Individual-patient monitoring in clinical practice: are available health status surveys adequate? Qual Life Res. 1995;4(4):293-307.

23. de Morton NA. The PEDro scale is a valid measure of the methodological quality of clinical trials: a demographic study. Aust J Physiother. 2009;55(2):129-33.

24. Musculoskeletal Health Sydney SoPHUoS: Physiotherapy evidence database (PEDro). 2018

25. Maher CG, Sherrington C, Herbert RD, Moseley AM, Elkins M. Reliability of the PEDro scale for rating quality of randomized controlled trials. Phys Ther. 2003;83(8):713-21.

26. Lundstrom M, Olofsson B, Stenvall M, Karlsson S, Nyberg L, Englund U, Borssen B, Svensson O, Gustafson Y. Postoperative delirium in old patients with femoral neck fracture: a randomized intervention study. Aging Clin Exp Res. 2007;19(3):178-86.

27. Stenvall M, Olofsson B, Lundstrom M, Englund U, Borssen B, Svensson NL, Gustafson Y. A multidisciplinary, multifactorial intervention program reduces postoperative falls and injuries after femoral neck fracture. Osteoporos Int. 2007:18(2):167-75

28. Stenvall M, Olofsson B, Nyberg L, Lundstrom M, Gustafson Y. Improved performance in activities of daily living and mobility after a multidisciplinary postoperative rehabilitation in older people with femoral neck fracture: a randomized controlled trial with 1-year follow-up. J Rehabil Med. 2007;39(3):232-8.

29. Stenvall M, Berggren M, Lundstrom M, Gustafson Y, Olofsson B. A multidisciplinary intervention program improved the outcome after hip fracture for people with dementia -- subgroup analyses of a randomized controlled trial. Arch Gerontol Geriatr. 2012;54(3):e284-9.

30. Prestmo A, Hagen G, Sletvold O, Helbostad JL, Thingstad P, Taraldsen K, Lydersen S, Halsteinli V, Saltnes T, Lamb SE, et al. Comprehensive geriatric care for patients with hip fractures: a prospective, randomised, controlled trial. Lancet. 2015;385:1623-33 North American Edition (9978).

31. Taraldsen K, Sletvold O, Thingstad P, Saltvedt I, Granat MH, Lydersen S, Helbostad JL. Physical behavior and function early after hip fracture surgery in patients receiving comprehensive geriatric care or orthopedic care--a randomized controlled trial. J Gerontol A Biol Sci Med Sci. 2014;69(3):338-45.

32. Abizanda $\mathrm{P}$, Leon $\mathrm{M}$, Dominguez-Martin $\mathrm{L}$, Lozano-Berrio $\mathrm{V}$, Romero $\mathrm{L}$, Luengo C, Sanchez-Jurado PM, Martin-Sebastia E. Effects of a short-term occupational therapy intervention in an acute geriatric unit. A randomized clinical trial. Maturitas. 2011;69(3):273-8.

33. Blanc-Bisson C, Dechamps A, Gouspillou G, Dehail P, Bourdel-Marchasson I. A randomized controlled trial on early physiotherapy intervention versus usual care in acute care unit for elderly: potential benefits in light of dietary intakes. J Nutr Health Aging. 2008;12(6):395-9.

34. Brown CJ, Foley KT, Lowman JD Jr, MacLennan PA, Razjouyan J, Najafi B, Locher J, Allman RM. Comparison of posthospitalization function and community mobility in hospital mobility program and usual care patients: a randomized clinical trial. JAMA Intern Med. 2016;176(7):921-7.

35. Czyzewski P, Szczepkowski M, Domaniecki J, Dabek A. Physiotherapy based on PNF concept for elderly people after conventional colon surgery. Pol Przegl Chir. 2013;85(9):475-82.

36. Eyres L, Unsworth CA. Occupational therapy in acute hospitals: the effectiveness of a pilot program to maintain occupational performance in older clients. Aust Occup Ther J. 2005;52(3):218-24.

37. Hagsten B, Svensson O, Gardulf A. Early individualized postoperative occupational therapy training in 100 patients improves ADL after hip fracture: a randomized trial. Acta Orthop Scand. 2004;75(2):177-83. 
38. Haines TP, O'Brien L, Mitchell D, Bowles K-A, Haas R, Markham D, Plumb S, Chiu T, May K, Philip K, et al. Study protocol for two randomized controlled trials examining the effectiveness and safety of current weekend allied health services and a new stakeholder-driven model for acute medical/surgical patients versus no weekend allied health services. Trials. 2015;16(1):133.

39. Jeffs KJ, Berlowitz DJ, Grant S, Lawlor V, Graco M, de Morton NA, Savige JA, Lim WK. An enhanced exercise and cognitive programme does not appear to reduce incident delirium in hospitalised patients: a randomised controlled trial. BMJ Open. 2013;3(6):e002569.

40. Jones CT, Lowe AJ, MacGregor L, Brand CA, Tweddle N, Russell DM. A randomised controlled trial of an exercise intervention to reduce functional decline and health service utilisation in the hospitalised elderly. Australas J Ageing. 2006;25(3):126-33.

41. Kimmel L, Liew S, Sayer J, Holland A. HIP4Hips (high intensity physiotherapy for HIP fractures in the acute hospital setting): a randomised controlled trial. Med J Aust. 2016;205(2):73-8.

42. Nikolaus T, Specht-Leible N, Bach M, Oster P, Schlierf G. A randomized trial of comprehensive geriatric assessment and home intervention in the care of hospitalized patients. Age Ageing. 1999;28(6):543-50.

43. Oldmeadow LB, Edwards ER, Kimmel LA, Kipen E, Robertson VJ, Bailey MJ. No rest for the wounded: early ambulation after hip surgery accelerates recovery. ANZ J Surg. 2006;76(7):607-11.

44. Siebens $\mathrm{H}$, Aronow H, Edwards D, Ghasemi Z. A randomized controlled trial of exercise to improve outcomes of acute hospitalization in older adults. J Am Geriatr Soc. 2000;48(12):1545-52.

45. Torres-Sanchez I, Valenza MC, Cabrera-Martos I, Lopez-Torres I, BenitezFeliponi A, Conde-Valero A. Effects of an exercise intervention in frail older patients with chronic obstructive pulmonary disease hospitalized due to an exacerbation: a randomized controlled trial. COPD. 2016;14(1):37-42.

46. Asplund K, Gustafson Y, Jacobsson C, Bucht G, Wahlin A, Peterson J, Blom JO, Angquist KA. Geriatric-based versus general wards for older acute medical patients: a randomized comparison of outcomes and use of resources. J Am Geriatr Soc. 2000;48(11):1381-8.

47. Barnes DE, Palmer RM, Kresevic DM, Fortinksy RH, Kowal J, Chren M-M Landefeld CS. Acute care for elders units produced shorter hospital stays at lower cost while maintaining Patients' functional status. Health Aff (Millwood). 2012;31(6):1227-36.

48. Counsell SR, Holder CM, Liebenauer LL, Palmer RM, Fortinsky RH, Kresevic DM, Quinn LM, Allen KR, Covinsky KE, Landefeld CS. Effects of a multicomponent intervention on functional outcomes and process of care in hospitalized older patients: a randomized controlled trial of acute Care for Elders (ACE) in a community hospital. J Am Geriatr Soc. 2000;48(12): $1572-81$.

49. Huusko TM, Karppi P, Avikainen V, Kautiainen H, Sulkava R. Randomised, clinically controlled trial of intensive geriatric rehabilitation in patients with hip fracture: subgroup analysis of patients with dementia. BMJ. 2000; 321(7269):1107-11.

50. Landefeld CS, Palmer RM, Kresevic DM, Fortinsky RH, Kowal J. A randomized trial of care in a hospital medical unit especially designed to improve the functional outcomes of acutely ill older patients. N Engl J Med. 1995; 332(20):1338-44.

51. Naglie G, Tansey C, Kirkland JL, Ogilvie-Harris DJ, Detsky AS, Etchells E, Tomlinson G, O'Rourke K, Goldlist B. Interdisciplinary inpatient care for elderly people with hip fracture: a randomized controlled trial. CMAJ. 2002; 167(1):25-32.

52. Pitkala KH, Laurila JV, Strandberg TE, Kautiainen $H$, Sintonen $H$, Tilvis RS. Multicomponent geriatric intervention for elderly inpatients with delirium: effects on costs and health-related quality of life. J Gerontol A Biol Sci Med Sci. 2008;63A(1):56-61.

53. Vidan M, Serra JA, Moreno C, Riquelme G, Ortiz J. Efficacy of a comprehensive geriatric intervention in older patients hospitalized for hip fracture: a randomized, controlled trial. J Am Geriatr Soc. 2005;53(9):1476-82

54. He M, Yu S, Wang L, Lv H, Qiu Z. Efficiency and safety of pulmonary rehabilitation in acute exacerbation of chronic obstructive pulmonary disease. Med Sci Monit. 2015;21:806-12.

55. Trials WGFOMC. Functional outcomes for clinical Trials in frail older persons: time to be moving. J Gerontol A Biol Sci Med Sci. 2008;63(2):160-4.

56. Organization $\mathbf{W H}$. The international classification of functioning, disability and health. Geneva: World Health Organization; 2001.

57. Buurman BM, Van Munster BC, Korevaar JC, De Haan RJ, De Rooij SE. Variability in measuring (instrumental) activities of daily living functioning and functional decline in hospitalized older medical patients: a systematic review. J Clin Epidemiol. 2011;64(6):619-27.

58. Terwee CB, Bot SDM, de Boer MR, van der Windt DAWM, Knol DL, Dekker J, Bouter LM, de Vet HCW. Quality criteria were proposed for measurement properties of health status questionnaires. J Clin Epidemiol. 2007;60(1):34-42.

59. Chung J, Demiris G, Thompson HJ. Instruments to assess mobility limitation in community-dwelling older adults: a systematic review. J Aging Phys Act. 2015;23(2):298-313.

60. Macri EM, Lewis JA, Khan KM, Ashe MC, De Morton NA. The de Morton mobility index: normative data for a clinically useful mobility instrument. J Aging Res. 2012;2012:353252.

61. Rush KL, Ouellet LL, Hautman MA. An analysis of elderly Clients' views of mobility. West J Nurs Res. 1998;20(3):295-311.

62. Soares Menezes KVR, Auger C, de Souza Menezes WR, Guerra RO. Instruments to evaluate mobility capacity of older adults during hospitalization: a systematic review. Arch Gerontol Geriatr. 2017;72:67-79.

63. de Morton NA, Berlowitz DJ, Keating JL. A systematic review of mobility instruments and their measurement properties for older acute medical patients. Health Qual Life Outcomes. 2008;6:44.

64. McCullagh R, Brady NM, Dillon C, Horgan NF, Timmons S. A review of the accuracy and utility of motion sensors to measure physical activity of frail, older hospitalized patients. J Aging Phys Act. 2016;24(3):465-75.

65. Erdem E, Tao Fout B, Korda HO, Abolude A. Hospital readmission rates in Medicare. J Hosp Adm. 2014:3:4.

66. Smet M. Cost characteristics of hospitals. Soc Sci Med. 2002;55(6):895-906.

67. OECD. Average length of stay in hospitals. In: Health at a glance 2017: OECD indicators. Paris, https://doiorg/101787/health_glance-2017-64-en: OECD Publishing; 2017. p. 176-7.

68. Marengoni A, Corrao S, Nobili A, Tettamanti M, Pasina L, Salerno F, lorio A, Marcucci M, Bonometti F, Mannucci PM. In-hospital death according to dementia diagnosis in acutely ill elderly patients: the REPOSI study. Int J Geriatr Psychiatry. 2011;26(9):930-6.

69. Masso Guijarro P, Aranaz Andres JM, Mira JJ, Perdiguero E, Aibar C. Adverse events in hospitals: the patient's point of view. Qual Saf Health Care. 2010; 19(2):144-7.

70. Schneider EC. Measuring mortality outcomes to improve health care: rational use of ratings and rankings. Med Care. 2002;40(1):1-3.

\section{Publisher's Note}

Springer Nature remains neutral with regard to jurisdictional claims in published maps and institutional affiliations.

\section{Ready to submit your research? Choose BMC and benefit from:}

- fast, convenient online submission

- thorough peer review by experienced researchers in your field

- rapid publication on acceptance

- support for research data, including large and complex data types

- gold Open Access which fosters wider collaboration and increased citations

- maximum visibility for your research: over $100 \mathrm{M}$ website views per year

At BMC, research is always in progress.

Learn more biomedcentral.com/submissions 